\title{
High Density Reflection Spectroscopy - II. The density of the inner black hole accretion disc in AGN
}

\author{
Jiachen Jiang ${ }^{\oplus}, 1,2 \star$ Andrew C. Fabian ${ }^{\oplus},{ }^{1}$ Thomas Dauser ${ }^{\oplus},{ }^{3}$ Luigi Gallo, ${ }^{4}$ \\ Javier A. García, ${ }^{3,5}$ Erin Kara, ${ }^{6}$ Michael L. Parker ${ }^{\oplus}{ }^{7}$ John A. Tomsick, ${ }^{8}$ \\ Dominic J. Walton ${ }^{1}$ and Christopher S. Reynolds ${ }^{1}$ \\ ${ }^{1}$ Institute of Astronomy, University of Cambridge, Madingley Road, Cambridge CB3 OHA, UK \\ ${ }^{2}$ Tsinghua Center for Astrophysics, Tsinghua University, Beijing 100084, China \\ ${ }^{3}$ Dr. Karl Remeis-Observatory and Erlangen Centre for Astroparticle Physics, Sternwartstr. 7, D-96049 Bamberg, Germany \\ ${ }^{4}$ Department of Astronomy and Physics, Saint Mary's University, 923 Robie Street, Halifax, NS B3H 3C 3, Canada \\ ${ }^{5}$ Cahill Center for Astronomy and Astrophysics, California Institute of Technology, Pasadena, CA 91125, USA \\ ${ }^{6}$ Department of Astronomy, University of Maryland, College Park, MD 20742-2421, USA \\ ${ }^{7}$ European Space Agency (ESA), European Space Astronomy Centre (ESAC), E-28691 Villanueva de la Cañada, Spain \\ ${ }^{8}$ Space Sciences Laboratory, 7 Gauss Way, University of California, Berkeley, CA 94720-7450, USA
}

Accepted 2019 August 17. Received 2019 August 14; in original form 2019 June 3

\begin{abstract}
We present a high density disc reflection spectral analysis of a sample of 17 Seyfert 1 galaxies to study the inner disc densities at different black hole mass scales and accretion rates. All the available XMM-Newton observations in the archive are used. OM observations in the optical/UV band are used to estimate their accretion rates. We find that 65 per cent of sources in our sample show a disc density significantly higher than $n_{\mathrm{e}}=10^{15} \mathrm{~cm}^{-3}$, which was assumed in previous reflection-based spectral analyses. The best-fitting disc densities show an anticorrelation with black hole mass and mass accretion rate. High density disc reflection model can successfully explain the soft excess emission and significantly reduce inferred iron abundances. We also compare our black hole spin and disc inclination angle measurements with previous analyses.
\end{abstract}

Key words: accretion, accretion discs-black hole physics-galaxies: Seyfert-X-rays: galaxies.

\section{INTRODUCTION}

High density disc reflection spectroscopy allows the density of the inner disc to be a free parameter during the analysis of the disc reflection spectra of accreting black hole (BH) systems. ${ }^{1}$ Previously, a fixed disc density of $n_{\mathrm{e}}=10^{15} \mathrm{~cm}^{-3}$ was commonly assumed. However the density of the inner disc is expected to be higher than this value in the less massive supermassive BHs (SMBHs, $M_{\mathrm{BH}} / \mathrm{M}_{\odot}$ $\left.=m_{\mathrm{BH}} \approx 10^{6}\right)$ and stellar-mass BHs $\left(m_{\mathrm{BH}}=4-20\right)$, as predicted by the standard Shakura \& Sunyaev (1973) disc model where all the disc energy is radiated away locally. It has been suggested that a high fraction of disc energy has to be dissipated in the corona instead of being radiated away at the disc surface in order to account for the typical spectral energy distribution of a Seyfert galaxy (Haardt \& Maraschi 1991). Theoretically, one will then obtain an even

\footnotetext{
^E-mail: jj447@cam.ac.uk

${ }^{1}$ We refer interested readers to the first paper of this series (Jiang et al. 2019b) for more introduction of disc reflection spectra of accreting BHs.
}

denser disc than the standard Shakura \& Sunyaev (1973) model after considering fraction of disc energy that is transferred to the corona (Svensson \& Zdziarski 1994). There have also been increasing observational indications for disc densities higher than $10^{15} \mathrm{~cm}^{-3}$ in both BH X-ray binaries (XRBs; e.g. Tomsick et al. 2018; Jiang et al. 2019b) and active galactic nuclei (AGN; e.g. Jiang et al. 2018; Garcia et al. 2019).

Most reflection simulations calculate spectra from a constant density slab parallel to a thin disc (e.g. Ross \& Fabian 1993; García \& Kallman 2010). One side of the slab is illuminated and heated up by coronal emission. These simulations then calculate the reflected spectra from the slab by considering both absorption and emission processes. The left-hand panel of Fig. 1 shows the temperature profiles of an illuminated ionized slab under hydrostatic equilibrium (García et al. 2016). At $n_{\mathrm{e}}=10^{15} \mathrm{~cm}^{-3}$ and $\xi=50 \mathrm{erg} \mathrm{cm} \mathrm{s}^{-1}$, the surface temperature of the ionized slab is about $2 \times 10^{5} \mathrm{~K}$. The temperature remains constant at larger depths before it drops to a lower temperature at Thomson depth $\tau_{\mathrm{T}}=0.1$. The drop in the temperature profile is because the recombination (cooling) process dominates over the heating process at $\tau_{\mathrm{T}}>0.1$. 

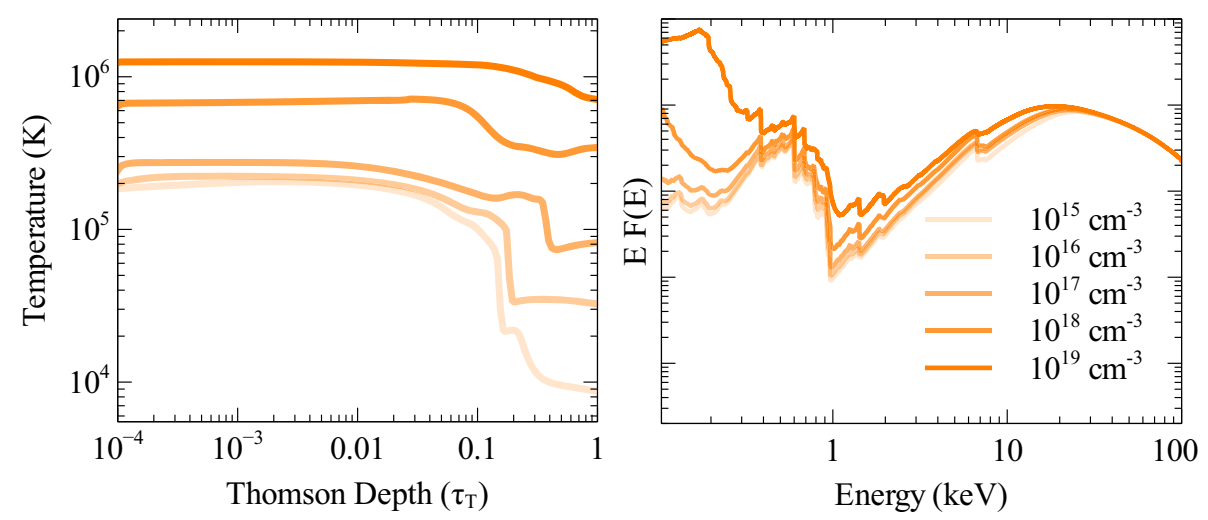

Figure 1. Left-hand panel: Temperature profiles of an illuminated slab under hydrostatic equilibrium for different electron densities calculated by García et al. (2016). Right-hand panel: Relativistic disc reflection spectra calculated by relxilld for different densities corresponding to the left-hand panel. A disc ionization parameter of $\xi=50$, emissivity index of $q=3$, maximum $\mathrm{BH}$ spin, and viewing angle of $i=30^{\circ}$ are assumed.

The electron density of the slab changes the temperature profile and thus affects the resulting reflection spectrum. For example, the temperature within $0.1 \tau_{\mathrm{T}}$ increases by a factor of 10 when $\log \left(n_{\mathrm{e}}\right)$ increases $^{2}$ from 15 to 19 . The temperature at $\tau_{\mathrm{T}}>0.1$ increases by an even larger factor. See the left-hand panel of Fig. 1 for temperature profiles for different electron densities. The change of temperature with density is because low energy photons in the disc are trapped by free-free absorption which increases the temperature of the slab at a high electron density (Ross \& Fabian 2007; García et al. 2016). Consequently, the reflected emission in the soft Xray band turns into a quasi-blackbody spectrum. See the right-hand panel of Fig. 1 for corresponding spectra for different disc densities.

In the first paper of this series, we demonstrate the effects of a high density model on the spectrum of the BH XRB GX $339-4$. The X-ray spectra of Seyfert galaxies and BH XRBs share many similarities, such as broad Fe K emission line and Compton hump above $10 \mathrm{keV}$ (e.g. Walton et al. 2012). Additionally, the soft Xray band of Seyfert galaxies is known to show variable excess emission ('soft excess'; e.g. Arnaud et al. 1985). The blackbodyshaped feature in a high density model may be able to explain this soft excess emission (e.g. Mallick et al. 2018; Garcia et al. 2019). The strongest supporting evidence for the reflection interpretation of the soft excess is the X-ray reverberation lags that have been found in various sources (e.g. Fabian et al. 2009; Zoghbi et al. 2012; De Marco et al. 2013; Kara et al. 2016).

In this paper, we fit high density models to the XMM-Newton spectra of a sample of Seyfert 1 galaxies. By conducting photometry with XMM-Newton OM observations, we are able to estimate the mass accretion rate for each $\mathrm{BH}$, and thus compare disc density with BH mass and accretion rate. In Section 2, we introduce our source sample and data reduction; in Section 3, we introduce the analysis method for EPIC-pn and OM observations; in Sections 4 and 5, we briefly summarize and discuss our results in this work and the first paper of this series. In Appendix A, we present a short introduction and details of the EPIC-pn spectral analysis for each individual source. In Appendix B, we present an estimation of the disc density at an optical depth of $\tau<1$ of a gas pressure-dominated disc in order to explain the disc densities in previous analyses of BH XRBs. High density disc reflection modelling for the extremely variable

${ }^{2}$ The value of $n_{\mathrm{e}}$ is reported in the unit of $\mathrm{cm}^{-3}$ hereafter. narrow-line Seyfert 1 galaxies 1H0707 - 495 will be presented in a future paper of this series.

\section{SAMPLE AND DATA REDUCTION}

We select 17 Seyfert 1 galaxies for our work. They include all 13 Seyfert 1 galaxies in the AGN Black Hole Mass Database (Bentz \& Katz 2015) that have been observed by XMM-Newton and 4 Seyfert 1 galaxies where broad $\mathrm{Fe} \mathrm{K} \alpha$ emission line was found and supersolar iron abundance was required in previous spectral analyses. The Seyfert 1 galaxies in our sample show no multiple warm absorbers or heavy obscuration along the line of sight. Therefore, we are able to have a clear view of the soft Xray emission from the innermost regions of their AGN. We ignore the Seyfert 2 galaxies in the AGN Black Hole Mass Database as they are mostly obscured in the X-ray band (Risaliti, Maiolino \& Salvati 1999). Table 1 shows the basic information for each source.

For BHs without a reverberation-mapping mass in the AGN Black Hole Mass Database (Bentz \& Katz 2015), we quote the mass measurement from $\mathrm{H} \beta$ line width (Bianchi et al. 2009). Due to the unknown geometry of the broad line region (BLR), the relation between $\mathrm{H} \beta$ line width and BLR velocity distribution has a large uncertainty. For example, Kaspi et al. (2000) assume a spherical BLR with an isotropic velocity distribution and predict $v_{\mathrm{BLR}}=\frac{\sqrt{3}}{2} \mathrm{FWHM}(\mathrm{H} \beta)$. But McLure \& Dunlop (2004) assume a disc-shaped BLR which predicts a velocity $\sqrt{3}$ times larger and a black hole mass three times larger than the values given by Kaspi et al. (2000). For this reason, we consider a conservative uncertainty of $\Delta \log \left(m_{\mathrm{BH}}\right)= \pm \log (3) \approx \pm 0.5$. The left-hand panel of Fig. 2 shows the $\mathrm{BH}$ mass distribution in our sample. A total of 75 per cent of sources in the sample have a $\mathrm{BH}$ mass between log $\left(m_{\mathrm{BH}}\right)=6-8$.

We consider all the available archival XMM-Newton observations for each AGN. A complete list of the XMM-Newton observations considered in this work is in Table A1. We extract EPIC-pn products using SAS 16.1.0, after filtering intervals dominated by flaring particle background. The task EPATPLOT is used to test for any pile-up effects. An annulus-shaped source region is used to extract source products if there is evidence for pile-up. The inner radius of the annulus is chosen to keep distribution patterns consistent with the model curves given by EPATPLOT. Background products are 
Table 1. The sample of Seyfert 1 galaxies considered in this work. The Galactic column density and extinction values are as calculated by Willingale et al. (2013). The source distances are from NED. The sixth column shows the total EPIC-pn exposure after filtering flaring particle background. $L_{\mathrm{X}}$ is the averaged Galactic absorption-corrected luminosity in the $0.5-10 \mathrm{keV}$ band. The upper table shows all the optical reverberation mapped Seyfert 1 galaxies in the AGN Black Hole Mass Database (Bentz \& Katz 2015). The lower table shows a sample of Seyfert 1 galaxies with $\mathrm{BH}$ mass measurements obtained by measuring $\mathrm{H} \beta$ line width (Bianchi et al. 2009). ${ }^{1}$ UGC 6728 only has MOS observations as its pn observation is dominated by flaring background.

\begin{tabular}{|c|c|c|c|c|c|c|}
\hline Name & $\log \left(M_{\mathrm{BH}} / \mathrm{M}_{\odot}\right)$ & $N_{\mathrm{H}}\left(10^{20} \mathrm{~cm}^{-2}\right)$ & $E(B-V)$ & $D(\mathrm{Mpc})$ & Expo (ks) & $\log \left(L_{X}\right)$ \\
\hline Ark 120 & $8.07_{-0.06}^{+0.05}$ & 14.5 & 0.126 & 148 & 504 & $44.338 \pm 0.002$ \\
\hline Mrk 1310 & $6.21_{-0.09}^{+0.07}$ & 2.66 & 0.029 & 86.7 & 35 & $41.67_{-0.03}^{+0.05}$ \\
\hline Mrk 279 & $7.44_{-0.13}^{+0.10}$ & 1.72 & 0.018 & 129 & 113 & $43.960_{-0.124}^{+0.008}$ \\
\hline Mrk 590 & $7.57_{-0.07}^{+0.06}$ & 2.93 & 0.031 & 107 & 36 & $43.143 \pm 0.012$ \\
\hline Mrk 79 & $7.61_{-0.14}^{+0.11}$ & 6.73 & 0.071 & 94.3 & 100 & $43.384_{-0.013}^{+0.012}$ \\
\hline NGC 4748 & $6.41_{-0.18}^{+0.11}$ & 4.07 & 0.052 & 65.5 & 26 & $43.033 \pm 0.011$ \\
\hline PG 0804 + 761 & $8.74 \pm 0.05$ & 3.51 & 0.032 & 475 & 32 & $44.728 \pm 0.009$ \\
\hline UGC 6728 & $5.85_{-0.36}^{+0.19}$ & 6.74 & 0.068 & 29.3 & $8.4,8.8^{1}$ & $42.053_{-0.019}^{+0.015}$ \\
\hline $1 \mathrm{H} 1934-063$ & $6.5 \pm 0.5$ & 19.5 & 0.278 & 42.5 & 105 & $43.037 \pm 0.006$ \\
\hline Ark 564 & $6.2 \pm 0.5$ & 6.74 & 0.068 & 106 & 402 & $43.972 \pm 0.006$ \\
\hline Swift J2127.4 + 5654 & $7.2 \pm 0.5$ & 91.4 & 1.532 & 61.1 & 326 & $43.380_{-0.009}^{+0.005}$ \\
\hline Ton S180 & $6.9 \pm 0.5$ & 1.54 & 0.016 & 263 & 148 & $44.01_{-0.09}^{+0.07}$ \\
\hline
\end{tabular}
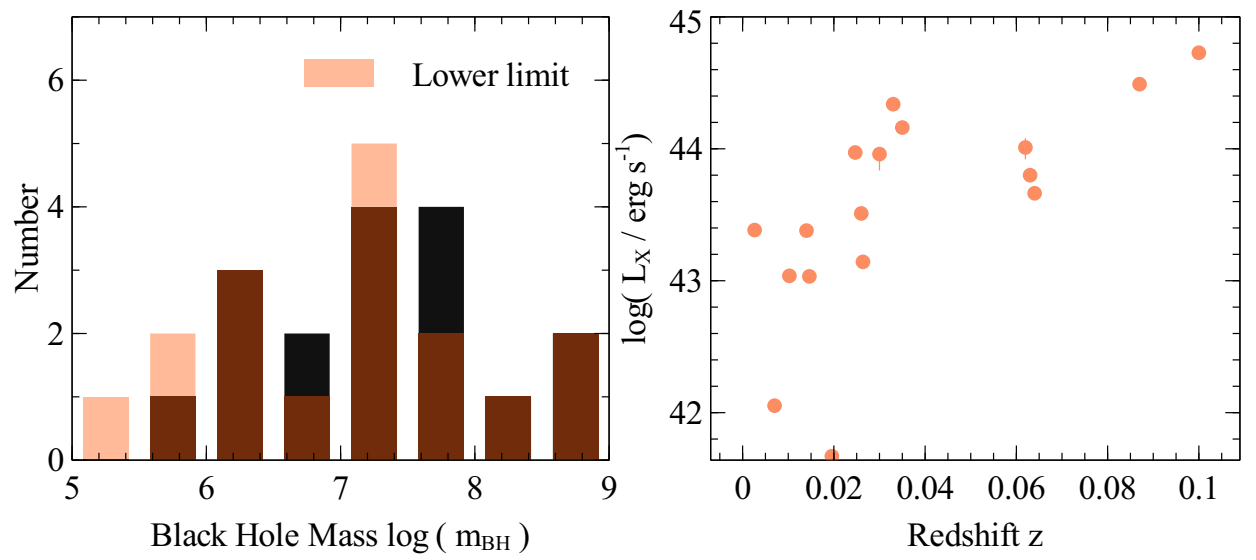

Figure 2. Left-hand panel: black hole mass distribution in our sample. The orange bars show the distribution of the lower limits of mass measurements. The brown bars show the overlaps of orange and black bars. Right-hand panel: source redshift and averaged X-ray luminosity distribution. The luminosities are calculated in the XMM-Newton band $(0.5-10 \mathrm{keV})$ after correcting for the Galactic absorption.

extracted from a nearby region on the same chip, avoiding the areas dominated by background $\mathrm{Cu} \mathrm{K}$ emission lines from the underlying electronics. We concentrate on the EPIC-pn spectra between 0.5 and $10 \mathrm{keV}$ due to its higher effective area compared to the two EPIC-MOS instruments. For UGC 6728, we extract EPIC-MOS spectra as its pn observation is dominated by flaring background. All the spectra are grouped to have a minimum signal-to-noise of 6 and oversample by a factor of 3 . Net pn exposures and averaged Xray luminosities after correcting for Galactic absorption are shown in the last column of Table 1. We stacked spectra from different observations for each source using ADDSPEC. In the right-hand panel of Fig. 2, we present the distribution of the averaged X-ray luminosities in the full pn band and source redshifts of our sample.

$X M M-N e w t o n$ Optical Monitor (OM) data are extracted using the task OMICHAIN, and the count rates are converted to Galactic extinction-corrected flux for each filter. The flux conversion factors are provided by SAS Watchout Website. ${ }^{3}$ The Galactic extinction curve calculated by Pei $(1992)$ is used to convert $E(B-V)$ to extinction at another wavelength.

\footnotetext{
${ }^{3}$ https://www.cosmos.esa.int/web/xmm-newton/sas-watchout-uvflux
} 


\section{XMM-Newton DATA ANALYSIS}

In this section, we first introduce the EPIC-pn spectral analysis using a variable density disc reflection model. Secondly, we estimate the $\mathrm{BH}$ mass accretion rates by measuring the source flux at an optical band with OM.

\subsection{EPIC-pn spectral analysis and disc density measurement}

The X-ray data analysis software XSPEC (Arnaud 1996) is used for broad-band spectral analysis of EPIC-pn data. C-stat (Cash 1979) is used. The model tbabs in XSPEC is used to account for the Galactic absorption. Galactic column densities $N_{\mathrm{H}}$ calculated by Willingale et al. (2013) are used and fixed during the spectral fitting. The values of Galactic column density can be found in Table 1 .

First, we fit EPIC-pn spectra with an absorbed power-law model in the $2-3$ and $8-10 \mathrm{keV}$ band, ignoring the iron band and the soft excess. Corresponding ratio plots are shown in Fig. 3. All the AGN in our sample show very strong soft excesses below 2 or $3 \mathrm{keV}$. Most AGN show evidence for emission features in the iron band. Some sources show a combination of broad and narrow emission lines.

Secondly, we model the soft excess and the broad emission line in the iron band using the relativistic reflection model relxilld (v1.2.0, García et al. 2016). relxilld combines the convolution model relconv (Dauser et al. 2013) and the illuminated ionized disc reflection model xillverd (García et al. 2016). The relconv model calculates the relativistic effects and corresponding emissivity profiles for emission lines in the reflection spectrum. A broken power-law emissivity profile (inner index $q_{1}$, outer index $q_{2}$, threshold radius $R_{\mathrm{r}}$ ) is assumed. In cases where $q_{2}$ and $R_{\mathrm{r}}$ are not constrained, we assume a simple power-law emissivity profile instead. The disc reflection model xillverd allows the disc density parameter to vary between $\log \left(n_{\mathrm{e}}\right)=15-19$. The solar abundance in xillverd is provided by Grevesse, Noels \& Sauval (1996). The ionization parameter $\xi$ is defined as $\xi=4 \pi F / n$ in unit of $\operatorname{erg~} \mathrm{cm} \mathrm{s}^{-1}$. The iron abundance $\left(Z_{\mathrm{Fe}}\right)$ is a free parameter during our spectral fitting. The reflection model xillver in the same model package is used to account for the narrow emission line feature if shown in the iron band. A simple power-law model is used to model the coronal emission. The convolution model $\mathrm{Cflux}$ is used to calculate the flux of each component in the $0.5-10 \mathrm{keV}$ band. An empirical reflection fraction $\left(F_{\text {refl }} / F_{\mathrm{pl}}\right)$ is used for simplicity and future comparison with other reflection models (e.g. reflionx, Ross \& Fabian 2007). In summary, the following models are used in XSPEC:

(i) tbabs * (cflux * relxilld + cflux * powerlaw $+\operatorname{cflux}^{*}$ xillver) (MODEL1) for sources that show narrow Fe $\mathrm{K}$ emission line in the iron band.

(ii) tbabs $*$ (cflux $*$ relxilld + cflux * powerlaw) (MODEL2) for sources that do not show any evidence for narrow $\mathrm{Fe} \mathrm{K}$ emission line.

(iii) tbabs * (cflux * relxilld + cflux $*$ powerlaw + zgauss + zgauss) (MODEL3) for sources that show complex ionized narrow emission lines in the iron band (e.g. Ark 120, Matt et al. 2014; Nardini et al. 2016).

(iv) tbabs * ABSORBER * (cflux * relxilld + cflux * powerlaw + cflux * xillver) (MODEL 4 ) for sources that show a thin warm absorber (e.g. Mrk 335, Longinotti et al. 2013; Parker et al. 2014) or a little obscuration (e.g. Swift J2127.4 + 5654, Miniutti et al. 2009). ABSORBER stands for the model that accounts for absorptions. For example, the ionized absorption model warmabs (Kallman \& Bautista 2001) is used to model warm absorber and ztbabs is used to model neutral obscuration.

\subsection{OM photometry and mass accretion rate}

The same method as in Raimundo et al. (2012) is used to determine the BH mass accretion rate with XMM-Newton OM observations, assuming a simple disc model with a steady accretion rate $\dot{M}$ and isotropic emission. The mass accretion rate is given by the following equation:

$\dot{M}=1.53{\frac{v L_{v}}{10^{45} \cos (i)}}^{3 / 2} \frac{10^{8}}{m_{\mathrm{BH}}}\left(\mathrm{M}_{\odot} \mathrm{yr}^{-1}\right)$

where $L_{v}$ is the luminosity in an optical band $v$ in $\operatorname{erg~s}^{-1}$ and $i$ is the viewing angle, which is obtained in the disc reflection analysis in Section 3.1. As explained in Raimundo et al. (2012), the lower energy band is less affected by a change of BH spin. For this reason, we choose $B$ band $(4500 \AA)$ as the priority wavelength for the calculation, as in Raimundo et al. (2012). If a source has no observation in $B$ band in the $X M M-N e w t o n$ archive, we choose the lowest energy band for calculation. Notice that $L_{\nu}$ has been corrected for Galactic extinction using the Galactic extinction curve calculated by Pei (1992) for each source. Table 2 shows the observed flux $F_{v}$ in the optical band named in the second column and corresponding mass accretion rate $\dot{m}=\dot{M} / \dot{M}_{\text {Edd }}$, where $\dot{M}_{\text {Edd }}$ is the Eddington accretion rate.

Note that we did not calculate the accretion rate by calculating the bolometric luminosity due to the large uncertainty of the bolometric conversion factor for the X-ray band flux (Vasudevan \& Fabian 2007) and the accretion efficiency in AGN (Raimundo et al. 2012).

\section{RESULTS}

Appendix A presents the details of EPIC-pn spectral analysis for individual sources in our sample. The best-fitting parameters are reported in Table A2 and the best-fitting models with corresponding best-fitting ratio plots are shown in Figs A1-A3. In this section, we summarize the results of the disc density measurements and compare our results with previous work.

\subsection{Disc densities in Seyfert 1 galaxies}

We present the best-fitting disc density values versus BH masses in the left-hand panel of Fig. 4. No obvious correlation between disc densities and $\mathrm{BH}$ masses in our sample is found. However, only upper limits of disc densities are achieved for sources with $\log \left(m_{\mathrm{BH}}\right)$ $>8$. This matches the previous analysis of another Seyfert 1 galaxy $1 \mathrm{H} 0419-577$ where the mass of the central BH is estimated to be $\log \left(m_{\mathrm{BH}}\right) \approx 8$ and a disc density of $\log \left(n_{\mathrm{e}}\right)<15.2$ is obtained with 90 per cent confidence (Jiang et al. 2019a). Disc densities significantly higher than $\log \left(n_{\mathrm{e}}\right)=15$ are found in AGN with $\log \left(m_{\mathrm{BH}}\right)<7$. By comparing the best-fitting disc densities with $\mathrm{BH}$ masses, we find that the assumption for a fixed disc density of $\log \left(n_{\mathrm{e}}\right)=15$ is mostly appropriate for SMBH with $\log \left(m_{\mathrm{BH}}\right)>8$ while a larger disc density is required for $\mathrm{SMBH}$ with $\log \left(m_{\mathrm{BH}}\right)<7$.

A second factor that changes the disc density is the $\mathrm{BH}$ mass accretion rate. Shakura \& Sunyaev (1973) predicts that the disc density of a radiation pressure-dominated disc and the mass accretion rate has the following relation $\log \left(n_{\mathrm{e}}\right) \propto-2 \log (\dot{m})$. The right-hand panel of Fig. 4 presents the disc density solution given by equation (8) in Svensson \& Zdziarski (1994), where $f$ is the fraction of power released from the disc to the corona. When $f=0$, 

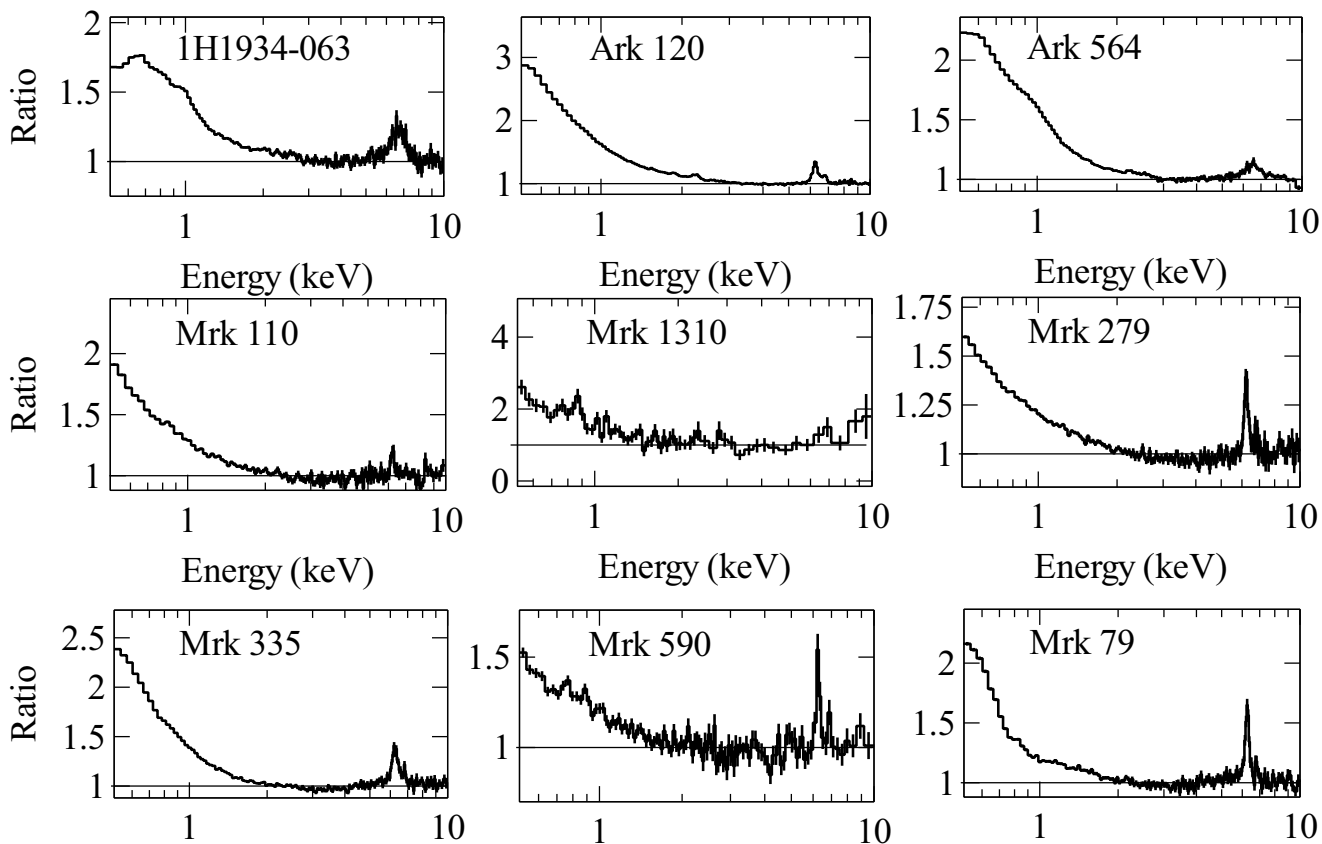

Energy (keV)
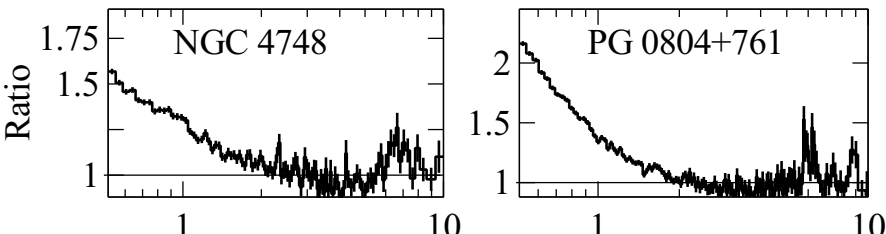

Energy (keV)

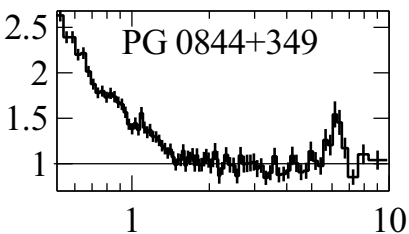

Energy (keV)

Energy (keV)
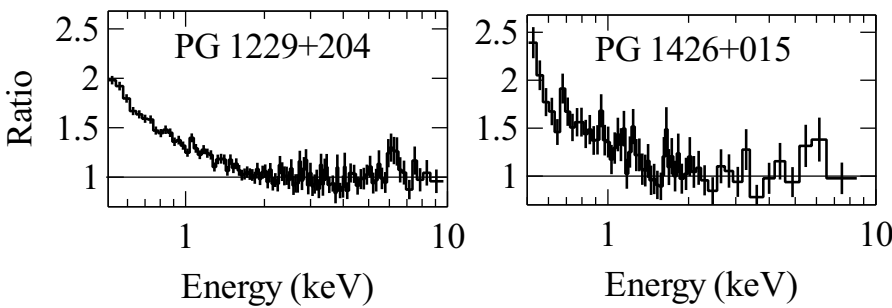

10

Energy $(\mathrm{keV})$

10

Energy (keV)
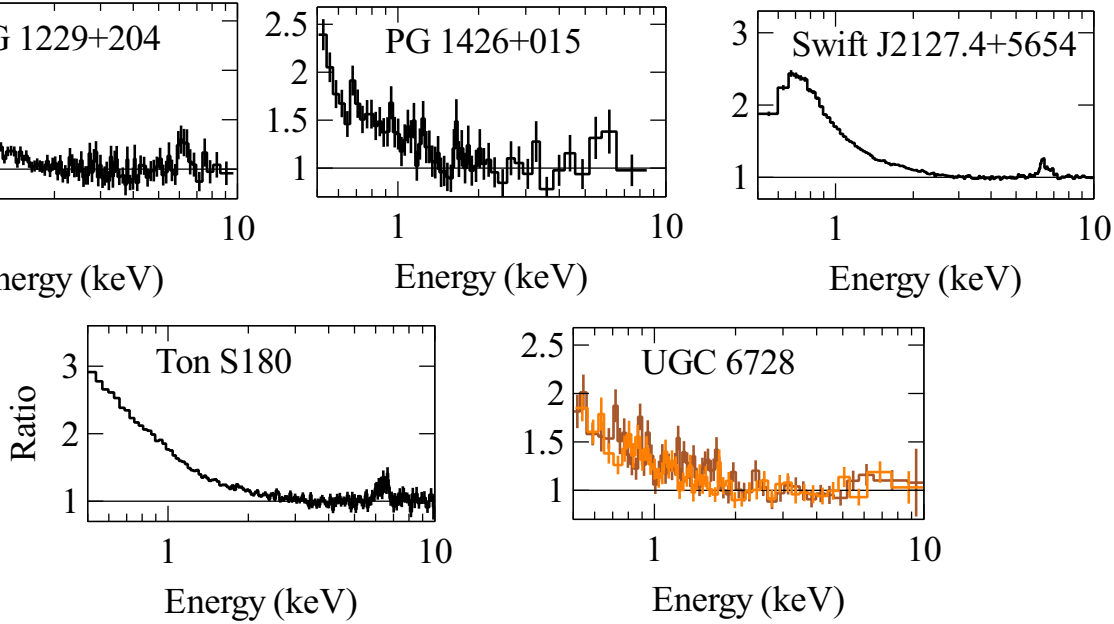

Figure 3. Ratio plots for pn spectra fitted with absorbed power-law models. MOS1 (orange) and MOS2 (brown) spectra are shown for UGC 6728 as its pn observation is dominated by flaring particle background.

this solution reproduces the results in Shakura \& Sunyaev (1973). The solid lines show the disc densities at $R=2 R_{\mathrm{g}}$ for different $f$, assuming: (1) a maximum BH spin; (2) the inner radius of the disc is $R_{\mathrm{ISCO}}$; (3) the conversion factor from radiative pressure to radiation flux in the radiative diffusion equation $\xi^{\prime}=1 .{ }^{4}$ The dotted

\footnotetext{
${ }^{4}$ The prime symbol is to distinguish the conversion factor from the disc ionization parameter $\xi$.
}

and dashed lines show the disc densities at $R=6,8 R_{\mathrm{g}}$ for $f=0.01$. The radius $R$ has less impact on the disc density than $f$, as shown in Fig. 4.

We present our results in the $\log \left(n_{\mathrm{e}}\right)$ versus $\log \left(m_{\mathrm{BH}} \dot{m}^{2}\right)$ diagram in the right-hand panel of Fig. 4. There is tentative evidence for an anticorrelation between $\log \left(n_{\mathrm{e}}\right)$ and $\log \left(m_{\mathrm{BH}} \dot{m}^{2}\right)$. We use a Monte Carlo approach to estimate the significance of the correlation. We assume $\log \left(n_{\mathrm{e}}\right)$ and $\log \left(m_{\mathrm{BH}} \dot{m}^{2}\right)$ follow normal distributions. A total of 100000 sets of points are drawn from distributions with the same 
Table 2. The flux of each source in an optical band measured by performing photometry with XMM-Newton OM observations. $F_{v}$ is the observed flux in the unit of $10^{-16} \mathrm{erg} \mathrm{s}^{-1} \mathrm{~cm}^{-2} \AA^{-1}$ in the band shown in the second column. The mass accretion rate $\dot{m}$ is in units of the Eddington accretion rate.

\begin{tabular}{lccc}
\hline Name & Band & $F_{v}$ & $\dot{m}$ \\
\hline 1H1934 & $\mathrm{U}$ & $59.8 \pm 0.3$ & $1.1_{-0.6}^{+2.3}$ \\
Ark 120 & $\mathrm{V}$ & $110.9 \pm 0.7$ & $0.76_{-0.08}^{+0.12}$ \\
Ark 564 & UVW1 & $71.7 \pm 0.6$ & $1.7_{-1.1}^{+1.7}$ \\
Mrk 110 & $\mathrm{B}$ & $72.8 \pm 0.4$ & $0.90_{-0.19}^{+0.23}$ \\
Mrk 1310 & $\mathrm{U}$ & $14.88 \pm 0.13$ & $0.6_{-0.10}^{+0.14}$ \\
Mrk 279 & $\mathrm{U}$ & $144.6 \pm 0.7$ & $0.75_{-0.16}^{+0.27}$ \\
Mrk 335 & $\mathrm{B}$ & $88.3 \pm 0.5$ & $0.74_{-0.07}^{+0.08}$ \\
Mrk 590 & $\mathrm{B}$ & $50.7 \pm 0.4$ & $0.31_{-0.05}^{+0.06}$ \\
Mrk 79 & $\mathrm{B}$ & $52.0 \pm 0.3$ & $0.13_{-0.07}^{+0.05}$ \\
NGC 4748 & $\mathrm{U}$ & $81.5 \pm 0.3$ & $2.2_{-0.5}^{+1.2}$ \\
PG 0804 & $\mathrm{V}$ & $105.3 \pm 0.3$ & $1.13_{-0.12}^{+0.15}$ \\
PG 0844 & $\mathrm{B}$ & $65.7 \pm 0.4$ & $1.20_{-0.4}^{+1.0}$ \\
PG 1229 & $\mathrm{UVM} 2$ & $69.9 \pm 0.8$ & $0.5_{-0.2}^{+0.4}$ \\
PG 1426 & $\mathrm{UVM} 2$ & $302.9 \pm 1.7$ & $0.28_{-0.06}^{+0.13}$ \\
Swift J2127 & $\mathrm{B}$ & $2.43 \pm 0.06$ & $1.0_{-0.2}^{+0.5}$ \\
Ton S180 & $\mathrm{U}$ & $49.3 \pm 0.5$ & $6_{-5}^{+12}$ \\
UGC 6728 & $\mathrm{U}$ & $53.7 \pm 0.4$ & $0.58_{-0.21}^{+0.76}$ \\
\hline
\end{tabular}

mean $\left(\right.$ mean $\left.\log \left(m_{\mathrm{BH}} \dot{m}^{2}\right)=7.1, \log \left(n_{\mathrm{e}}\right)=16.89\right)$ and deviation $\left(\sigma_{\log \left(m_{\mathrm{BH}} \dot{m}^{2}\right)}=0.92, \sigma_{\log \left(n_{\mathrm{e}}\right)}=1.17\right)$ of the sample. We find 11304 sets of points that exceed the Spearman correlation coefficient of our result $(-0.67)$. This gives 11 per cent probability of the observed correlation from randomly distributed points. By considering the large uncertainties of mass measurements, the significance of the correlation shown in Fig. 4 is even lower. The correlation between these two parameters is weak in our sample due to the following reasons: (1) the uncertainties of the BH mass measurements; (2) other physics, such as the vertical structure of the disc density and the fraction $(f)$ of power that is released from the disc to the coronal region. We will discuss the effect of $f$ on the disc density in Section 5 .

In summary, we find some evidence for a trend that high disc density is commonly seen in AGN that show low $m_{\mathrm{BH}} \dot{m}^{2}$ values. It is important to note that the Galactic BH GX 339-4 shows a similar pattern. The disc density of GX $339-4$ in the low flux-hard state is higher than the density in the high flux-soft state (Jiang et al. 2019b)

\subsection{Comparison with previous work}

\subsubsection{Disc iron abundances}

A super solar iron abundance was often seen in previous reflectionbased spectral analysis where a fixed disc density $\left(n_{\mathrm{e}}=10^{15} \mathrm{~cm}^{-3}\right)$ was assumed. A significant decrease of inferred iron abundance is found in our new disc reflection modelling. Fig. 5 presents the best-fitting disc iron abundances in this work shown in black and the best-fitting iron abundances in previous work in orange. Corresponding references are labelled on the right-hand side. This figure only includes the sources that have reflection analyses in previous published work. The disc iron abundances obtained by using a high density model for IRAS 13224 and GX 339 - 4 are from Jiang et al. (2018, 2019b).

Several sources in our sample show a significant decrease of inferred disc iron abundance when a high density disc reflection model is used. These sources are 1H 1934, Ton S180, IRAS 13224, and GX 339 - 4. A similar conclusion was found in Tomsick et al. (2018), where an iron abundance at the solar level is required for the intermediate state of the BH XRB Cyg X-1 when a high density model is used. Only PG 0844 shows a slightly higher disc iron abundance compared to previous results. However the abundance is still near the solar level $\left(Z_{\mathrm{Fe}}<3 \mathrm{Z}_{\odot}\right)$.

The decrease of the inferred iron abundances is due to the increase of the continuum in the reflection component when a high density model is used. For example, the best-fitting reflection fraction for Ton $\mathrm{S} 180$ is $f_{\text {refl }}=1.9 \pm 0.7$ when the spectrum is modelled using a variable density disc reflection model. ${ }^{5}$ The averaged spectrum of Ton S180 is dominated by the disc reflection component and requires an iron abundance of $Z_{\mathrm{Fe}}=3 \pm 2 \mathrm{Z}_{\odot}$. Instead of using a high density model, we fit the soft excess emission of Ton S180 with a soft cutoff model in addition to a $\log \left(n_{\mathrm{e}}\right)=15$ disc model, similar to the work of Parker, Miller \& Fabian (2018). Although such a model combination slightly improves the fit by $\Delta \mathrm{C}$-stat $=7$ with 1 more parameter, the disc reflection component with a fixed disc density requires a very high iron abundance of $Z_{\mathrm{Fe}}>8 \mathrm{Z}_{\odot}$. See Fig. 6 for comparison of best-fitting models for disc densities and Table A3 for best-fitting parameters when cut of $\mathrm{f}$ is used to model the soft excess. A much lower reflection fraction of $f_{\text {refl }}=0.70$ is required. Therefore in order to model the strong $\mathrm{Fe} \mathrm{K}$ emission feature in the iron band of Ton S180, a higher iron abundance is required.

Although a high density disc model is able to decrease the inferred iron abundance, some AGN still show a high iron abundance compared to solar (e.g. IRAS 13224 - 3809; Jiang et al. 2018). In future, we will further study the metallicity of these systems by allowing more element abundances to be free parameters during the spectral modelling.

\subsubsection{BH spins and disc inclination angles}

Fig. 7 shows disc inclination angles and $\mathrm{BH}$ spins obtained in our work in black and previous analyses in orange. Although we do not expect the orientation of an AGN and the spin of an SMBH to vary in different observations, we cannot rule out instrumental effects of different observations on the measurements (e.g. see discussion section of Brenneman 2013). Moreover, the X-ray continuum emission from the Seyfert AGN mentioned in Fig. 7 is known to be very variable (e.g. Frederick et al. 2018). The continuum modelling can potentially have an impact on the determination of the red and blue wings of their broad $\mathrm{Fe} \mathrm{K} \alpha$ emission lines and thus affect the measurements of $\mathrm{BH}$ spin and disc inclination angle, respectively.

Most of the disc inclination angles measured using high density disc reflection spectroscopy are consistent with previous results over the 90 per cent confidence ranges, except for 1H 1934, Ark 120, Swift J2127, IRAS 13224, and GX 339 - 4. We only obtain an upper limit of $n_{\mathrm{e}}$ for Ark 120. Therefore the difference of inclination angles for Ark 120 is unlikely due to the high density model. The

\footnotetext{
${ }^{5}$ Note that the reflection fraction $f_{\text {refl }}$ here is not the same reflection fraction used in Dauser et al. (2016). An empirical definition of $F_{\text {refl }} / F_{\mathrm{pl}}$ in the $X M M-N e w t o n$ band is used in order to quantify the relative strength of the reflection component compared to the coronal emission.
} 

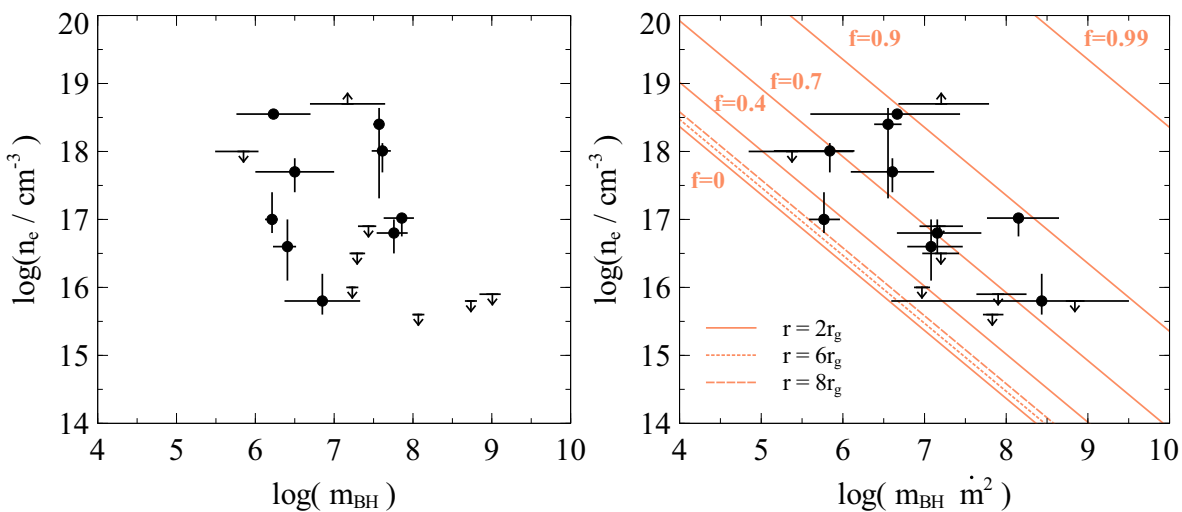

Figure 4. Left-hand panel: Disc density $\log \left(n_{\mathrm{e}}\right)$ versus BH mass $\log \left(m_{\mathrm{BH}}\right)$. Only upper limits of disc density are obtained for black holes with log $\left(m_{\mathrm{BH}}\right)>$ 8 , indicating a lower disc density in high- $m_{\mathrm{BH}}$ AGN. Right-hand panel: Disc density $\log \left(n_{\mathrm{e}}\right)$ versus $\log \left(m_{\mathrm{BH}} \dot{m}^{2}\right)$. The solid orange lines are the solutions for disc density at $r=2 r_{\mathrm{g}}$ for different values of $f$ (Svensson \& Zdziarski 1994). The inner radius is assumed to be at $R_{\mathrm{ISCO}}$ of a maximally spinning BH ( $R_{\text {in }}=$ $1 r_{\mathrm{g}}$ ). The dotted and dashed straight lines are for $r=6,8 r_{\mathrm{g}}$ and $f=0$.

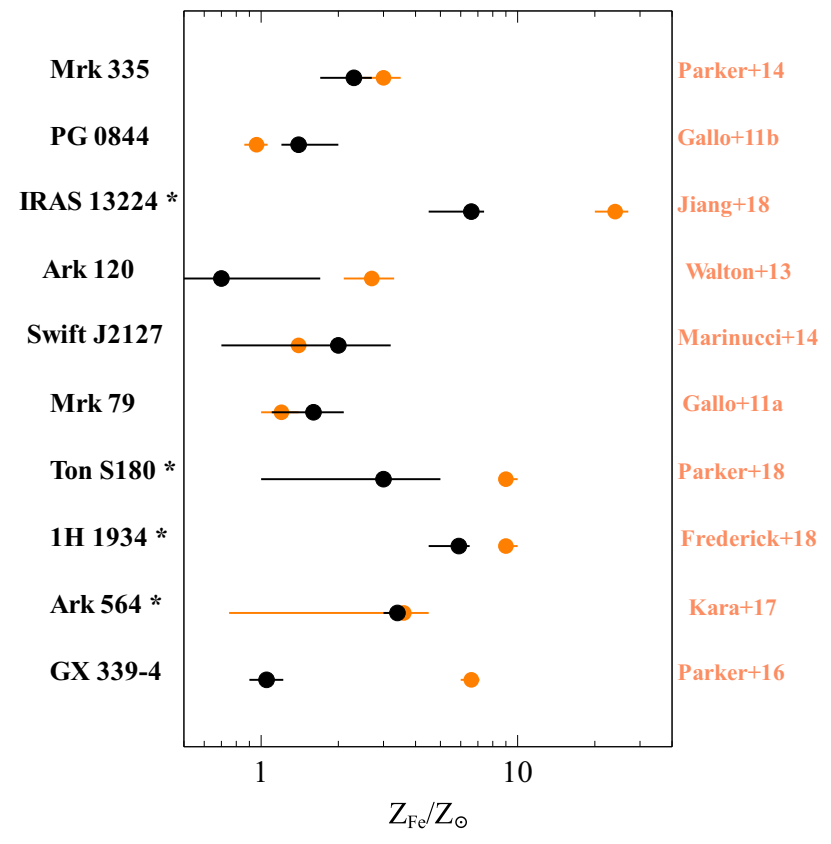

Figure 5. Best-fitting iron abundances $Z_{\mathrm{Fe}}$ obtained by modelling with a variable density model shown in black compared with previous results shown in orange where $\log \left(n_{\mathrm{e}}\right)=15$ was assumed. Corresponding references are labelled on the right-hand side. * An additional model component is used for soft excess in previous analyses for these AGN.

other four sources all show higher inferred inclination angles when a variable density model is used. Although the disc inclination angle of GX 339 - 4 using a high disc density model is higher than the result in Wang-Ji et al. (2018) and lower than the result in Parker et al. (2016), our measurement is still consistent with previous reflection-based analyses within a $2 \sigma$ uncertainty range. See the discussion section of Jiang et al. (2019b). We also notice that Tomsick et al. (2018) found a different conclusion when using a high density model for Cyg $\mathrm{X}-1$. A significantly smaller disc inclination angle is found for this source when a variable density model is used.

Most of the best-fitting BH spins for our sample given by high density disc reflection spectroscopy are near the Kerr limit and consistent with previous analyses within 90 per cent confidence ranges, except for 1H 1934 and Ton S180. In previous analyses of 1H 1934 and Ton S180 (Frederick et al. 2018; Parker et al. 2018), an additional component was used to model the soft excess. In the previous section, we explain how a high density model changes the continuum modelling. For example, the power-law continuum of Ton $\mathrm{S} 180$ has $\Gamma=2.38$ when a high density model is used. By contrast, a harder continuum of $\Gamma=2$ is found for the same spectrum if a cut of $f$ model is added for soft excess. See Fig. 6 for comparison of two models. In this case, the spin is not constrained (see Table A3). By contrast, Nardini, Fabian \& Walton (2012) obtained a disc inner radius of $R_{\text {in }} \approx 2.4 r_{\mathrm{g}}$ by modelling the soft excess of Ton $\mathrm{S} 180$ with a second relativistic disc reflection model instead of a cutoff model and assuming $\log \left(n_{\mathrm{e}}\right)=15$. Their disc inner radius measurement is consistent with our spin measurement using a high density disc reflection model.

Similarly, Kara et al. (2017) claim that the spin of Ark 564 is not constrained by analysing its Suzaku and NuSTAR spectra while Walton et al. (2013) obtains a spin of $a_{*}=0.96_{-0.06}^{+0.01}$ for the same source by analysing its $S u z a k u$ observations. The latter measurement is consistent with our result. The difference in Kara et al. (2017) was that an additional thermal bremsstrahlung component was used to model the soft excess and the Suzaku XIS spectrum below $1 \mathrm{keV}$ was ignored. This work and Walton et al. (2013) fit the soft excess emission with disc reflection model.

Future simultaneous hard X-ray observations, such as from NUSTAR, will be helpful for constraining the continuum emission by including the reflection Compton hump above $10 \mathrm{keV}$. An example is the analysis of the simultaneous NUSTAR and XMM-Newton observation of the NLS1 Mrk 1044 in Mallick et al. (2018), where a high density disc reflection model of $\log \left(n_{\mathrm{e}}\right) \approx 16$ explains the $0.5-78 \mathrm{keV}$ band spectrum of this source with no requirement for an additional component.

\section{DISCUSSION AND CONCLUSIONS}

We summarize the disc density measurements in this paper and previous analyses (Jiang et al. 2018, 2019b; Mallick et al. 2018; Tomsick et al. 2018; Garcia et al. 2019) in Fig. 8. The results from previous work of AGN are marked by orange points. The blue circles are for GX 339-4 observations in 2015 and the blue squares are for observations in 2013. The black points are the measurements for our sample. The orange straight lines are the same as the ones 

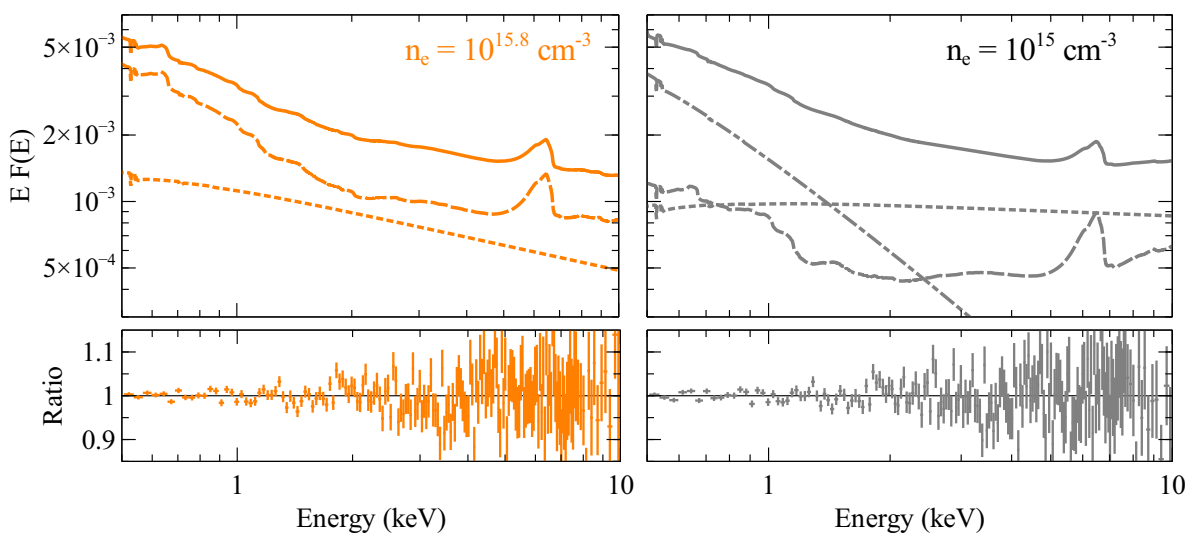

Figure 6. Left-hand panel: The best-fit models for Ton S180 when a high density disc model is used and the corresponding ratio plot. Solid: total model; dashed: high density disc model with $\log \left(n_{\mathrm{e}}\right)=15.6$; dotted: power-law continuum. The unit of the y-axis of the upper panel is keV cts $\mathrm{cm}^{-2} \mathrm{~s}^{-1}$. Right-hand panel: Same as the left-hand panel but an additional soft cutoff model (dash-dotted line) is used to model the soft excess. A fixed disc density of log $\left(n_{\mathrm{e}}\right)$ $=15$ is assumed. In this model, the coronal emission shows a harder continuum and the reflection component makes less contribution to the total emission compared to the models in the left-hand panel.
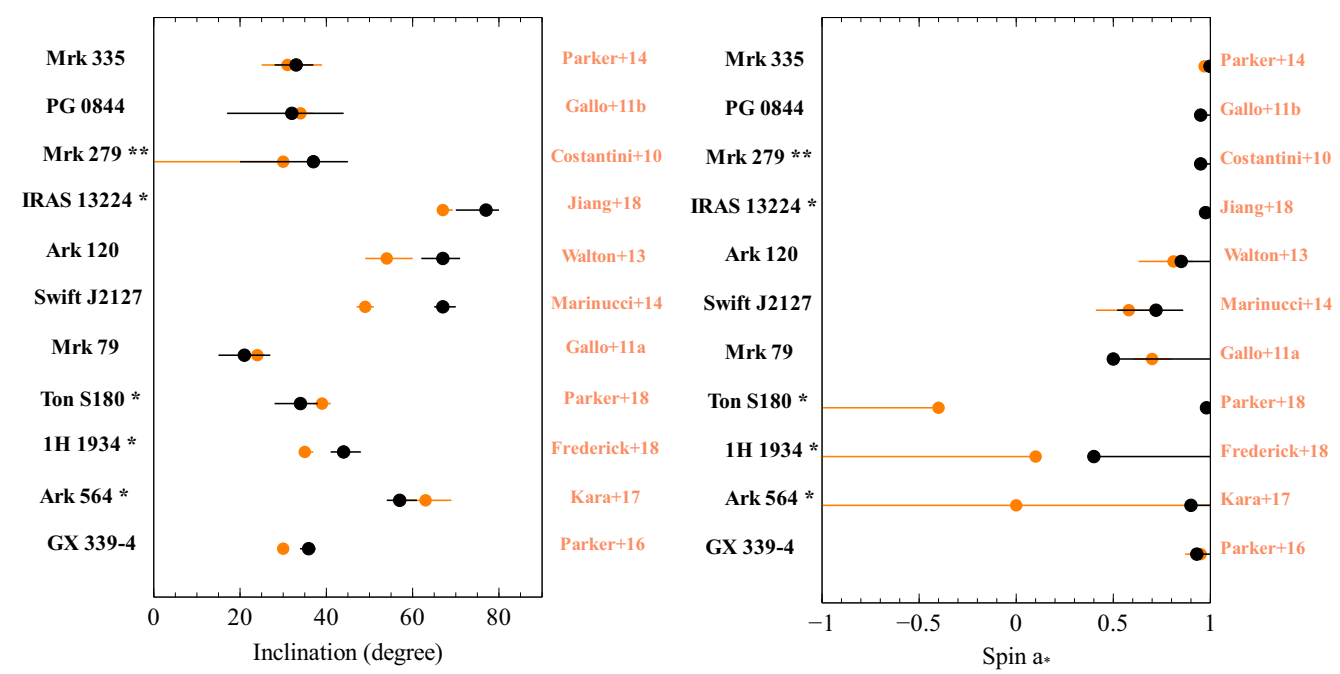

Figure 7. Left-hand panel: Comparison of disc inclination angles obtained by modelling with a variable density model shown in black and previous results shown in orange where $\log \left(n_{\mathrm{e}}\right)=15$ was assumed. Right-hand panel: Comparison of BH spins. ${ }^{*}$ An additional model component is used for soft excess in previous analyses for these AGN. ** Costantini et al. (2010) only obtained the inclination angle of Mrk 279 by modelling the broad iron line with the disc emission line model laor, assuming a maximum $\mathrm{BH}$ spin.

as in Fig. 4 and the blue straight line is the solution for $f=0, R=$ $2 R_{\mathrm{g}}$, and $\xi^{\prime}=2$. We can draw the following conclusions from this diagram:

First, a significantly higher disc density is found in stellar-mass BHs than in SMBHs. A total of 65 per cent of SMBHs in our sample show evidence for a disc density significantly higher than $\log \left(n_{\mathrm{e}}\right)$ $=15$. In the hard state of GX 339-4 and the intermediate state of Cyg X-1, the density of the disc is at least $\log \left(n_{\mathrm{e}}\right)>20.5$. In the high flux state of GX 339-4, a disc density of $\log \left(n_{\mathrm{e}}\right) \approx 19$ is required, similar with the disc density in AGN with $\log \left(m_{\mathrm{BH}} \dot{m}^{2}\right)<7$.

Secondly, the accretion rate affects the disc density in the same way as the BH mass: a higher disc density is found where the $\mathrm{BH}$ accretes at a lower fraction of its Eddington limit. This conclusion has been found previously by studying the different states of the $\mathrm{BH}$ XRB GX 339 - 4 (Jiang et al. 2019b) where only the accretion rate can be changing. Similarly, we find tentative evidence for a similar conclusion for AGN. For example, Mrk 509 and PG $0804+761$ have a similar BH mass of $m_{\mathrm{BH}} \approx 10^{8}$ (Bentz \& Katz 2015; Garcia et al. 2019). However, Mrk 509 has a higher disc density than PG $0804+761$. This might be due to the higher accretion rate in PG $0804+761(\dot{m} \approx 1.0-1.3$; see Table 2$)$ than in Mrk 509 ( $\dot{m}=0.2-0.4$; Petrucci et al. 2013).

Thirdly, theoretically most coronal heating mechanisms assume that a large fraction of the disc energy is dissipated in the coronal region (e.g. magnetic coronae model; Galeev, Rosner \& Vaiana 1979; Coroniti 1981; Stella \& Rosner 1984). Assuming $\xi^{\prime}=1$, our analysis suggests at least 10 per cent of the power in the disc is released to the corona in AGN. If a higher value of $\xi^{\prime}$ (e.g. $\xi^{\prime}$ $=2$; Svensson \& Zdziarski 1994) is assumed, an even higher $f$ is expected. See the blue and orange solid lines in Fig. 8.

Fourthly, although there is a weak correlation between $\log \left(n_{\mathrm{e}}\right)$ and $\log \left(m_{\mathrm{BH}} \dot{m}^{2}\right)$ in our sample, a disc density of $\log \left(n_{\mathrm{e}}\right)>$ 16 is clearly found in AGN with $\log \left(m_{\mathrm{BH}} \dot{m}^{2}\right)<6.5$. The weak correlation could be due to other uncertain effects, such as different $f$, 


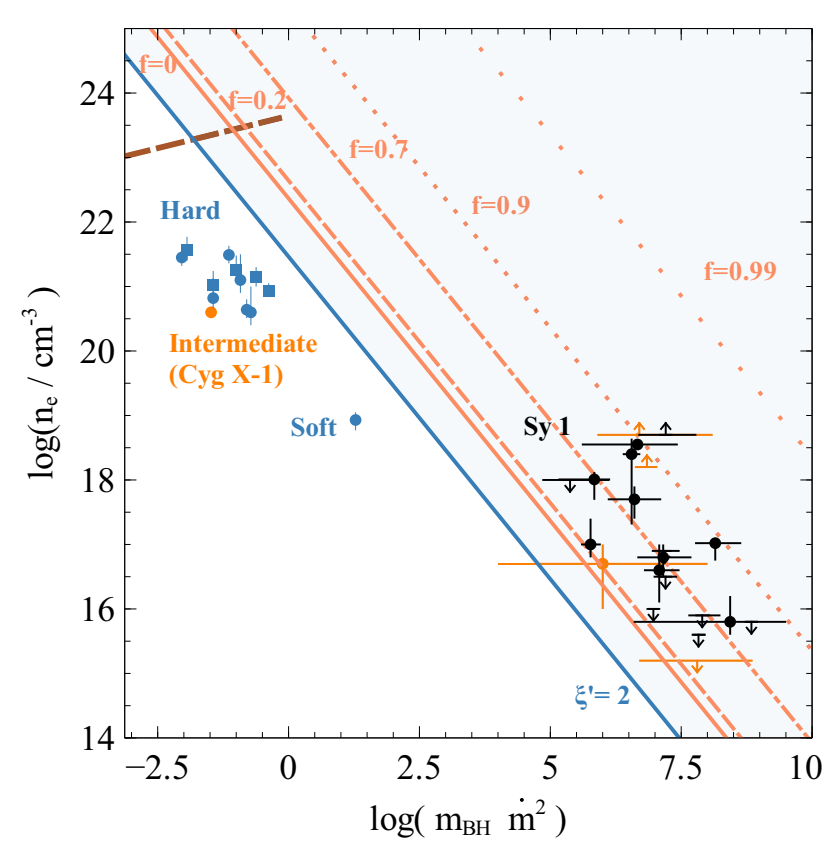

Figure 8. Disc density $\log \left(n_{\mathrm{e}}\right)$ versus $\log \left(m_{\mathrm{BH}} \dot{m}^{2}\right)$ for our sample and previous analysis. Previous analysis are marked by orange points (see the text for references). The blue circles represent GX 339 - 4 observations in 2015 and the squares represent observations in 2013 that are analysed in Jiang et al. (2019b). A BH mass of $m_{\mathrm{BH}}=10$ is assumed for GX339 - 4. The orange lines are the same as the ones in Fig. 4. The blue solid line is the solution assuming $f=0, \xi^{\prime}=2$, and $r=2 r_{\mathrm{g}}$. The red dashed line shows the solution for a gas pressure-dominated disc at $r=2 r_{\mathrm{g}}$ assuming $f=0$. All the disc density curves are calculated by Svensson $\&$ Zdziarski (1994).

the vertical structure of the disc density in reality, or the uncertainties of the BH mass measurements.

Fifthly, the disc densities that our reflection model obtains for BH XRBs are significantly lower than the prediction of the standard thin disc model (the solid orange line in Fig. 8; Shakura \& Sunyaev 1973). Some potential explanations are: (1) the disc density parameter in the reflection model is the density in the surface of the disc while the thin disc models assume a uniform disc density in the vertical direction; (2) the $\mathrm{BH}$ mass and the distance measurements of GX $339-4$ are uncertain. However, we notice that the disc density of Cyg X-1 is still below the prediction, although the mass and the distance of $\mathrm{Cyg} X-1$ are well constrained in this case (Orosz et al. 2011; Reid et al. 2011); (3) the stellar-mass BH discs during the observations considered in Tomsick et al. (2018); Jiang et al. $(2019 \mathrm{~b})$ are likely to be dominated by gas pressure instead of radiation pressure with $L_{\mathrm{Bol}} \approx L_{\mathrm{X}}<4$ per cent $L_{\mathrm{Edd}}$. At such low luminosities, they correspond to the gas pressure-dominated disc regime according to Svensson \& Zdziarski (1994). Consequences of gas pressure-dominated discs happening during the state transition of $\mathrm{BH}$ transients will be addressed in future work. However, the densities measured in XRBs are still 10-100 times lower than the density of a gas pressure-dominated disc in Shakura \& Sunyaev (1973), which is shown by the red dashed line in Fig. 8. It suggests that more physics is needed to be considered, such as the vertical structure of a gas pressure-dominated disc. In Appendix B, we estimate the disc density within optical depth $\tau<1$ of a gas pressuredominated disc.

In conclusion, we find that the high density disc reflection model cannot only decrease the inferred iron abundance but also successfully explains the $0.5-10 \mathrm{keV}$ band spectra of the Seyfert 1 galaxies in our sample with no requirement for additional components for the soft excess emission. The density of the disc is significantly higher than the previous $\log \left(n_{\mathrm{e}}\right)=15$ assumption in AGN with $\log \left(m_{\mathrm{BH}} \dot{m}^{2}\right)<7.5$ in our sample. This is consistent with the prediction of the standard thin disc model (Shakura \& Sunyaev 1973; Svensson \& Zdziarski 1994). More future work is needed to complete the high density disc reflection spectroscopy for AGN in following approaches: (1) abundances of other elements in addition to iron need to be considered; (2) simultaneous broadband observations of these Seyfert galaxies are required (e.g. from NuSTAR) to test whether the high density model can account for the Compton hump above $10 \mathrm{keV}$; (3) time-resolved spectral analysis is required to study the short-term variability of the disc density within one observation.

\section{ACKNOWLEDGEMENTS}

JJ acknowledges support by the Cambridge Trust and the Chinese Scholarship Council Joint Scholarship Programme (201604100032). DJW acknowledges support from an STFC Ernest Rutherford Fellowship. ACF acknowledges support by the ERC Advanced Grant 340442. MLP is supported by European Space Agency (ESA) Research Fellowships. JAG acknowledges support from the Alexander von Humboldt Foundation. The authors are also grateful to Andrew J. Young, Paul C. Hewett, and Sergei Dyda for valuable discussion.

\section{REFERENCES}

Arnaud K. A., 1996, XSPEC: The First Ten Years. Astronomical Data Analysis Software and Systems V, College Park, MD

Arnaud K. A. et al., 1985, MNRAS, 217, 105

Bentz M. C., Katz S., 2015, PASP, 127, 67

Bianchi S., Guainazzi M., Matt G., Fonseca Bonilla N., Ponti G., 2009, A\&A, 495, 421

Boller T., Balestra I., Kollatschny W., 2007, A\&A, 465, 87

Brenneman L., 2013, Measuring the Angular Momentum of Supermassive Black Holes. Springer, Berlin

Cash W., 1979, ApJ, 228, 939

Coroniti F. V., 1981, ApJ, 244, 587

Costantini E., Kaastra J. S., Korista K., Ebrero J., Arav N., Kriss G., Steenbrugge K. C., 2010, A\&A, 512, A25

Dauser T., Garcia J., Wilms J., Böck M., Brenneman L. W., Falanga M., Fukumura K., Reynolds C. S., 2013, MNRAS, 430, 1694

Dauser T., García J., Walton D. J., Eikmann W., Kallman T., McClintock J., Wilms J., 2016, A\&A, 590, A76

De Marco B., Ponti G., Cappi M., Dadina M., Uttley P., Cackett E. M., Fabian A. C., Miniutti G., 2013, MNRAS, 431, 2441

Fabian A. C. et al., 2009, Nature, 459, 540

Frank J., King A., Raine D. J., 2002, Accretion Power in Astrophysics, 3rd, Springer, Berlin

Frederick S., Kara E., Reynolds C., Pinto C., Fabian A., 2018, ApJ, 867, 67

Galeev A. A., Rosner R., Vaiana G. S., 1979, ApJ, 229, 318

Gallo L. C., Miniutti G., Miller J. M., Brenneman L. W., Fabian A. C., Guainazzi M., Reynolds C. S., 2011a, MNRAS, 411, 607

Gallo L. C., Grupe D., Schartel N., Komossa S., Miniutti G., Fabian A. C., Santos-Lleo M., 2011b, MNRAS, 412, 161

Gallo L. C. et al., 2015, MNRAS, 446, 633

García J., Kallman T. R., 2010, ApJ, 718, 695

García J. A., Fabian A. C., Kallman T. R., Dauser T., Parker M. L., McClintock J. E., Steiner J. F., Wilms J., 2016, MNRAS, 462, 751

Garcia J. A. et al., 2019, ApJ, 871, 88 
Grevesse N., Noels A., Sauval A. J., 1996, in Holt S. S., Sonneborn G., eds, ASP Conf. Ser. Vol. 99, Cosmic Abundances. Astron. Soc. Pac., San Francisco. p. 117

Grupe D., Komossa S., Gallo L. C., 2007, ApJ, 668, L111

Grupe D., Komossa S., Gallo L. C., Longinotti A. L., Fabian A. C., Pradhan A. K., Gruberbauer M., Xu D., 2012, ApJS, 199, 28

Haardt F., Maraschi L., 1991, ApJ, 380, L51

Jiang J. et al., 2018, MNRAS, 477, 3711

Jiang J., Walton D. J., Fabian A. C., Parker M. L., 2019a, MNRAS, 483, 2958

Jiang J., Fabian A. C., Wang J., Walton D. J., García J. A., Parker M. L., Steiner J. F., Tomsick J. A., 2019b, MNRAS, 484, 1972

Kallman T., Bautista M., 2001, ApJS, 133, 221

Kara E., Fabian A. C., Cackett E. M., Uttley P., Wilkins D. R., Zoghbi A., 2013, MNRAS, 434, 1129

Kara E. et al., 2015, MNRAS, 446, 737

Kara E., Alston W. N., Fabian A. C., Cackett E. M., Uttley P., Reynolds C. S., Zoghbi A., 2016, MNRAS, 462, 511

Kara E., García J. A., Lohfink A., Fabian A. C., Reynolds C. S., Tombesi F., Wilkins D. R., 2017, MNRAS, 468, 3489

Kaspi S., Smith P. S., Netzer H., Maoz D., Jannuzi B. T., Giveon U., 2000, ApJ, 533, 631

Longinotti A. L., Bianchi S., Santos-Lleo M., Rodríguez-Pascual P., Guainazzi M., Cardaci M., Pollock A. M. T., 2007, A\&A, 470, 73

Longinotti A. L. et al., 2013, ApJ, 766, 104

Mallick L. et al., 2018, MNRAS, 479, 615

Marinucci A. et al., 2014, MNRAS, 440, 2347

Matt G. et al., 2014, MNRAS, 439, 3016

McLure R. J., Dunlop J. S., 2004, MNRAS, 352, 1390

Miniutti G., Panessa F., de Rosa A., Fabian A. C., Malizia A., Molina M., Miller J. M., Vaughan S., 2009, MNRAS, 398, 255

Nagao T., Murayama T., Taniguchi Y., 2001, ApJ, 546, 744

Nardini E., Fabian A. C., Walton D. J., 2012, MNRAS, 423, 3299

Nardini E., Porquet D., Reeves J. N., Braito V., Lobban A., Matt G., 2016, ApJ, 832, 45

Orosz J. A., McClintock J. E., Aufdenberg J. P., Remillard R. A., Reid M. J., Narayan R., Gou L., 2011, ApJ, 742, 84

Osterbrock D. E., Phillips M. M., 1977, PASP, 89, 251

Page K. L., Schartel N., Turner M. J. L., O’Brien P. T., 2004, MNRAS, 352, 523

Parker M. L. et al., 2014, MNRAS, 443, 1723

Parker M. L. et al., 2016, ApJ, 821, L6

Parker M. L., Miller J. M., Fabian A. C., 2018, MNRAS, 474, 1538

Pei Y. C., 1992, ApJ, 395, 130

Petrucci P. O. et al., 2013, A\&A, 549, A73

Porquet D. et al., 2019, A\&A, 623, A11

Raimundo S. I., Fabian A. C., Vasudevan R. V., Gandhi P., Wu J., 2012, MNRAS, 419, 2529

Reeves J. N., Porquet D., Braito V., Nardini E., Lobban A., Turner T. J., 2016, ApJ, 828, 98

Reid M. J., McClintock J. E., Narayan R., Gou L., Remillard R. A., Orosz J. A., 2011, ApJ, 742, 83

Risaliti G., Maiolino R., Salvati M., 1999, ApJ, 522, 157

Ross R. R., Fabian A. C., 1993, MNRAS, 261, 74

Ross R. R., Fabian A. C., 2007, MNRAS, 381, 1697

Shakura N. I., Sunyaev R. A., 1973, A\&A, 24, 337

Stella L., Rosner R., 1984, ApJ, 277, 312

Svensson R., Zdziarski A. A., 1994, ApJ, 436, 599

Takahashi H., Hayashida K., Anabuki N., 2010, PASJ, 62, 1483

Tomsick J. A. et al., 2018, ApJ, 855, 3

Vasudevan R. V., Fabian A. C., 2007, MNRAS, 381, 1235

Vaughan S., Boller T., Fabian A. C., Ballantyne D. R., Brandt W. N., Trümper J., 2002, MNRAS, 337, 247

Véron-Cetty M. P., Véron P., 2006, A\&A, 455, 773

Walton D. J., Reis R. C., Cackett E. M., Fabian A. C., Miller J. M., 2012, MNRAS, 422, 2510

Walton D. J., Nardini E., Fabian A. C., Gallo L. C., Reis R. C., 2013, MNRAS, 428, 2901
Wang-Ji J. et al., 2018, ApJ, 855, 61

Willingale R., Starling R. L. C., Beardmore A. P., Tanvir N. R., O’Brien P. T., 2013, MNRAS, 431, 394

Zoghbi A., Fabian A. C., Reynolds C. S., Cackett E. M., 2012, MNRAS, 422,129

\section{APPENDIX A: OBSERVATION AND SPECTRAL ANALYSIS DETAILS}

\section{A1 1H $1934-603$}

1H $1934-603$ is a narrow-line Seyfert 1 galaxy (NLS1, Nagao, Murayama \& Taniguchi 2001) that shows fast variability in the $\mathrm{X}$-ray band. Previously by analysing the archival XMM-Newton and NUSTAR observations of $1 \mathrm{H} 1934-603$, Frederick et al. (2018) discovered that the disc reflection component lags behind the coronal power-law continuum by $\approx 20 \mathrm{~s}$. By conducting a novel spectral analysis using a fixed disc density reflection model, a super solar iron abundance $\left(Z_{\mathrm{Fe}}>9 \mathrm{Z}_{\odot}\right)$ is required for the reflection spectral modelling (Frederick et al. 2018).

A ratio plot for an averaged EPIC-pn spectrum of 1H $1934-603$ against an absorbed power-law model is shown in Fig. 3. A broad emission line feature is visible in the iron band. By fitting the emission line with a simple Gaussian line model zgauss, we obtain a best-fitting rest-frame line energy at $E_{\text {line }}=6.65 \pm 0.05 \mathrm{keV}$ with $\sigma=0.49_{-0.07}^{+0.08} \mathrm{keV}$. The equivalent width (EW) of the emission line is $264_{-7}^{+8} \mathrm{eV}$. Small residuals are visible at $5 \mathrm{keV}$ when the line feature is modelled by a simple Gaussian line model, requiring a more physical modelling for the broad emission line (e.g. relativistic disc line). No obvious narrow line component has been found.

Based on the spectral analysis in the iron band, we then model the broad-band spectrum with MODEL2. MODEL2 can provide a very good fit with C-Stat $/ \nu=242.35 / 180$. The best-fitting model and corresponding ratio plot are shown in Fig. A1. No structural residuals are found in the ratio plot. A disc density of $\log \left(n_{\mathrm{e}}\right)=17.7_{-0.3}^{+0.2}$ is required with a disc iron abundance of $Z_{\mathrm{Fe}} / \mathrm{Z}_{\odot}=5.9_{-1.4}^{+0.6}$. The iron abundance obtained with the disc density as a free parameter is much lower than the value obtained in previous analysis (Frederick et al. 2018). We obtain a lower limit of the BH spin $a_{*}>0.4$, which is higher than the previous analysis ( $a_{*}<0.1$; Frederick et al. 2018). Note that the previous analysis in Frederick et al. (2018) models the soft excess emission with an additional blackbody model and assumes a fixed disc density at $\log \left(n_{\mathrm{e}}\right)=15$. In this work, we model both the soft excess emission and the broad iron emission line with only one disc reflection model by allowing the disc density to be a free parameter.

\section{A2 Ark 120}

Ark 120 is a nearby Seyert 1 galaxy (e.g. Osterbrock \& Phillips 1977) that is well-studied in the X-ray band. This source shows little or no evidence for X-ray absorption (e.g. Reeves et al. 2016). Previous spectral analysis of Ark 120 shows evidence for three line components in the iron band (Nardini et al. 2016). Two of the three line components are narrow emission lines, corresponding to a neutral $\mathrm{Fe} \mathrm{K} \alpha$ emission line and an ionized $\mathrm{Fe} \mathrm{K} \alpha$ emission line. The third line component is broader with FWHM $\approx 5000 \mathrm{~km} \mathrm{~s}^{-1}$.

A ratio plot for an averaged EPIC-pn spectrum of Ark 120 against an absorbed power-law model is shown in Fig. 3. A combination of narrow emission lines and a broad emission line is shown in the iron band. The line shapes are similar to these found in Nardini et al. (2016). By fitting the line features with three Gaussian 
Table A1. A list of all the XMM-Newton observations considered in this work. SW: small window mode; FF: full frame mode; LW: large window mode. Expo: Net exposure after correcting flaring particle background.

\begin{tabular}{|c|c|c|c|c|c|c|c|c|c|}
\hline Name & obs ID & Expo (ks) & Date & pn mode & Name & obs ID & Expo (ks) & Date & pn mode \\
\hline 1H1934 & 0761870201 & 93 & $2015-10-03$ & SW & - & 0306870101 & 90 & 2006-01-03 & SW \\
\hline & 0693781501 & 85 & 2013-02-18 & SW & - & 0600540501 & 53 & 2009-06-13 & $\mathrm{FF}$ \\
\hline & 0721600201 & 86 & 2014-03-18 & SW & - & 0600540601 & 76 & 2009-06-11 & FF \\
\hline & 0721600301 & 85 & 2014-03-20 & SW & - & 0741280201 & 36 & $2015-12-30$ & $\mathrm{FF}$ \\
\hline & 0721600401 & 85 & $2014-03-22$ & SW & Mrk 590 & 0109130301 & 7 & 2002-01-01 & SW \\
\hline \multirow[t]{6}{*}{ Ark 564} & 0006810101 & 7.4 & 2000-06-17 & SW & Mrk 79 & 0103862101 & 5.3 & 2000-10-09 & SW \\
\hline & 0006810301 & 7.0 & 2001-06-19 & SW & - & 0400070201 & 14 & 2006-09-30 & SW \\
\hline & 0206400101 & 69 & 2005-01-05 & SW & - & 0400070301 & 14 & 2006-09-30 & SW \\
\hline & 0670130201 & 41 & 2011-05-24 & SW & - & 0400070401 & 14 & 2007-03-19 & SW \\
\hline & 0670130301 & 39 & 2011-05-31 & SW & - & 0502091001 & 53 & 2008-04-26 & SW \\
\hline & 0670130401 & 38 & 2011-06-06 & SW & NGC 4748 & 0723100401 & 26 & 2014-01-14 & LW \\
\hline Mrk 110 & 0201130501 & 33 & 2004-11-15 & SW & PG 1426 & 0102040501 & 0.6 & $2000-07-28$ & $\mathrm{FF}$ \\
\hline Mrk 1310 & 0723100301 & 35 & 2013-12-09 & SW & UGC 6728 & 0312191601 & $8.4,8.8$ & $2006-02-23$ & $\mathrm{FF}$ \\
\hline \multirow[t]{4}{*}{ Mrk 279} & 0083960101 & 13 & 2002-05-07 & $\mathrm{FF}$ & Ton S180 & 0110890401 & 21 & $2000-12-14$ & SW \\
\hline & 0302480401 & 40 & 2005-11-15 & SW & - & 0110890701 & 13 & 2002-06-30 & SW \\
\hline & 0302480501 & 38 & $2005-11-17$ & SW & - & 0764170101 & 93 & 2015-07-03 & SW \\
\hline & 0302480604 & 22 & $2005-11-19$ & SW & - & 0790990101 & 21 & 2016-06-13 & SW \\
\hline \multirow[t]{5}{*}{ Swift J2127 } & 0601741901 & 5 & 2009-11-11 & $\mathrm{FF}$ & - & - & - & - & - \\
\hline & 0655450101 & 84 & $2010-11-29$ & SW & - & - & - & - & - \\
\hline & 0693781701 & 94 & 2012-11-04 & SW & - & - & - & - & - \\
\hline & 0693781801 & 93 & 2012-11-06 & SW & - & - & - & - & - \\
\hline & 0693781901 & 50 & $2012-11-08$ & SW & - & - & - & - & - \\
\hline
\end{tabular}

line model zgauss, we obtain two of the three line components are at $6.43_{-0.02}^{+0.05} \mathrm{keV}\left(\mathrm{EW}=38_{-12}^{+42} \mathrm{eV}\right)$ and $7.03_{-0.02}^{+0.03} \mathrm{keV}(\mathrm{EW}=$ $27_{-12}^{+20} \mathrm{eV}$ ). The best-fitting line widths for these two line components are $<0.01 \mathrm{keV}$ and $0.06_{-0.05}^{+0.04} \mathrm{keV}$ correspondingly. The $6.43 \mathrm{keV}$ emission line can be interpreted as the neutral $\mathrm{Fe} \mathrm{K} \alpha$ emission line and the other line can be interpreted as the hydrogenic iron. The third line component is located at $6.49_{-0.02}^{+0.05} \mathrm{keV}\left(\mathrm{EW}=100_{-13}^{+14}\right)$. The width of the line is $0.30_{-0.05}^{+0.06} \mathrm{keV}$, indicating a broad emission line from the inner disc region.

Based on the existence of both a neutral and ionized narrow iron emission lines, we model the broad-band spectrum with MODEL3. MODEL3 can provide a good fit with C-stat $/ \nu=288.61 / 168$. The best-fitting model and corresponding ratio plot are shown in Fig. A1. Only an upper limit of the disc density is found $\log \left(n_{\mathrm{e}}\right)<15.6$. A solar iron abundance is required for the spectral modelling. A fixed spin $a_{*}=0$ and a fixed viewing angle $i=30^{\circ}$ are assumed in Nardini et al. (2016). In contrast, we obtain a high black hole spin $a_{*}>0.85$ and a high viewing angle $i=67_{-5}^{+4^{\circ}}$, which are consistent with previous reflection-based analysis of Suzaku observations of the same source (e.g. $a_{*} \approx 0.81, i \approx 54^{\circ}$, Walton et al. 2013) and other spin measurement methods (e.g. Porquet et al. 2019).

\section{A3 Ark 564}

Ark 564 is a very variable NLS1 in the X-ray band. Detailed studies of its X-ray reverberation lags with $X M M$-Newton observations have been done in previous analyses (e.g. Kara et al. 2013). A high iron abundance $\left(Z_{\mathrm{Fe}} / \mathrm{Z}_{\odot} \approx 3\right)$ was obtained by analysing the simultaneous Suzaku and NuSTAR spectra above $1 \mathrm{keV}$ (Kara et al. 2017).

We present a broad-band spectral analysis of the averaged EPICpn spectrum of Ark 564 with a total net pn exposure of $402 \mathrm{ks}$. A ratio plot against an absorbed power-law model is shown in Fig. 3. A very strong emission line feature is shown in the iron band and a very strong soft excess is shown below $3 \mathrm{keV}$. Fitting the emission line in the iron band with zgauss offers a good fit with some remaining residuals at $5.5 \mathrm{keV}$, requiring more physical modelling (e.g. relativistic disc reflection model). The central energy of the line is at $E_{\text {line }}=6.59_{-0.07}^{+0.06} \mathrm{keV}$ in the source frame with a line width of $\sigma=0.44_{-0.08}^{+0.09} \mathrm{keV}$. The equivalent width of the best-fitting line model is $120_{-8}^{+12} \mathrm{eV}$. No obvious narrow emission line feature at $6.4 \mathrm{keV}$ is found in the iron band.

We fit the full band spectrum with MODEL2 due to the lack of evidence for narrow emission lines in the iron band. MODEL 2 offers a very good fit for the averaged EPIC-pn spectrum of Ark 564 with $\mathrm{C}$-stat $/ \nu=191.32 / 180$. The best-fitting model and corresponding ratio plot are shown in Fig. A1. A close-to-solar iron abundance is obtained and a very high disc density of $\log \left(n_{\mathrm{e}}\right)=18.55 \pm 0.07$ is required for the spectral fitting. No additional component is required to model the soft excess. A high BH spin of $a_{*}>0.9$ is found, similar to the previous analysis by analysing Suzaku observations of the same source ( $a_{*} \approx 0.96$; Walton et al. 2013). The BH spin parameter was however not constrained in Kara et al. (2017). An 

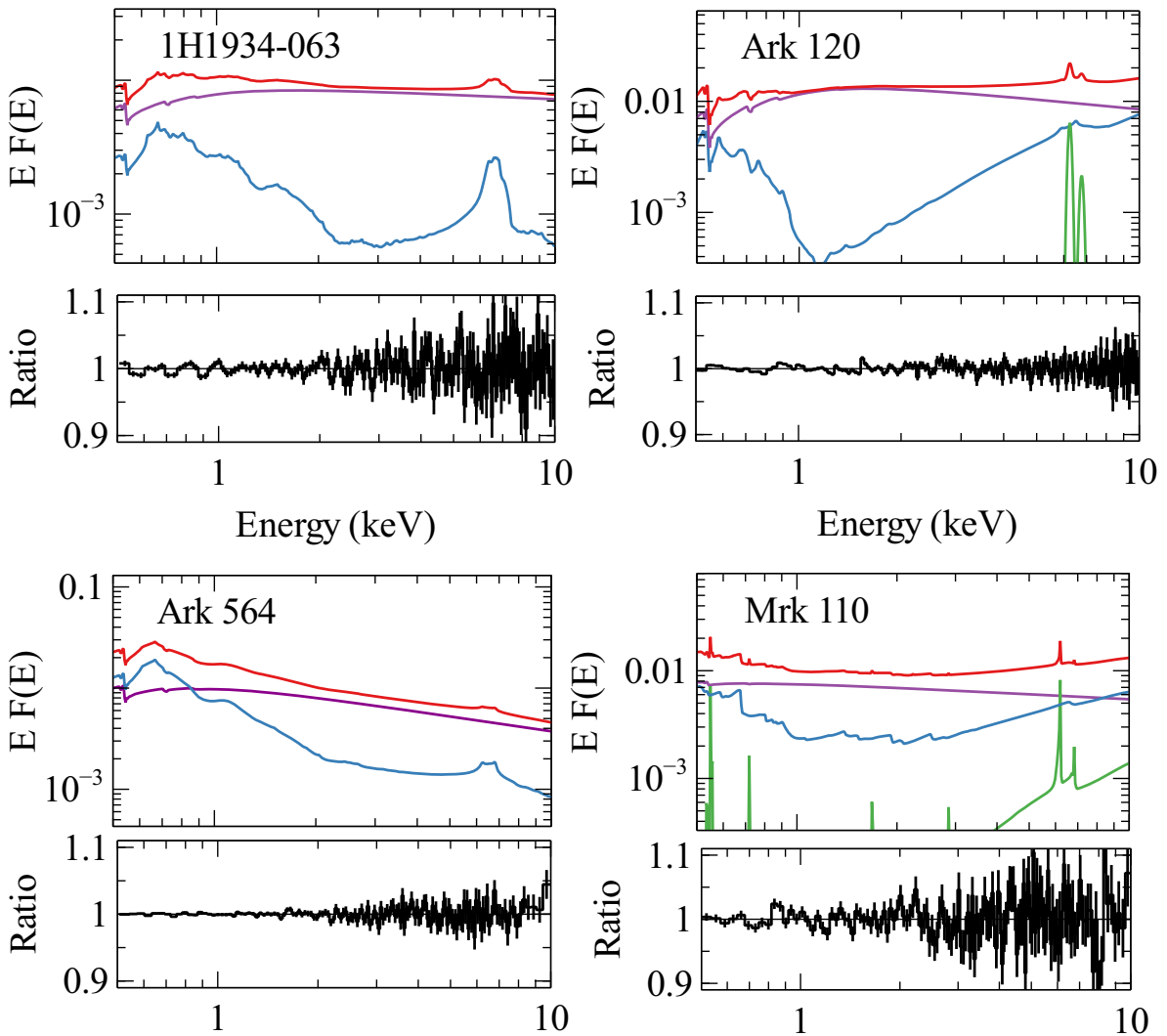

Energy $(\mathrm{keV})$

Energy (keV)

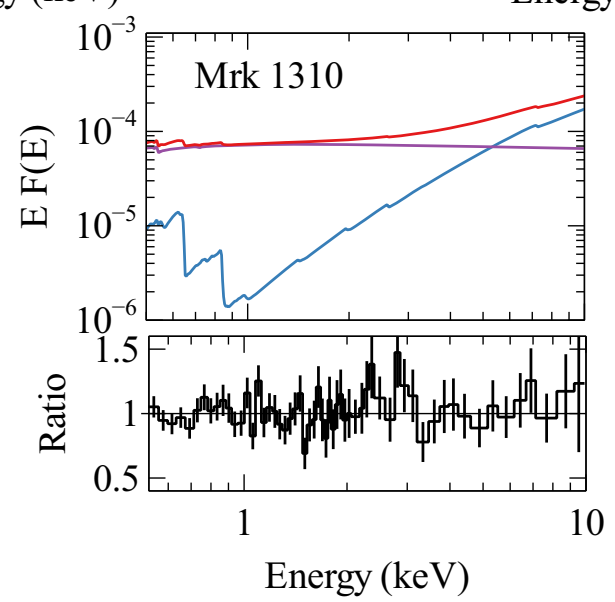

Figure A1. The best-fitting model and corresponding ratio plot for each source. Red: total model; blue: relativistic reflection model; green: distant reflector; purple: power-law shaped coronal emission. The unit of the $\mathrm{y}$-axis in the model plots is $\mathrm{keV} \mathrm{cts} \mathrm{cm} \mathrm{cm}^{-1}$.

inclination angle of $i=57_{-3}^{+4^{\circ}}$ is obtained, which is consistent with the results in Walton et al. (2013) and Kara et al. (2017).

\section{A4 Mrk 110}

Mrk 110 is an NLS1 (Véron-Cetty \& Véron 2006) and has been observed by XMM-Newton once for a net pn exposure of $33 \mathrm{ks}$. Boller, Balestra \& Kollatschny (2007) show a complete analysis of the RGS and EPIC spectra. Only a narrow Fe K emission line was found previously.

By fitting the EPIC-pn spectrum of the only XMM-Newton observation of Mrk 110, we confirm that only a narrow emission line is shown in the iron band. A ratio plot of the EPIC-pn spectrum fitted with an absorbed power-law model is shown in Fig. 3. By using a simple Gaussian line model zgauss, we obtain the best-fitting line width of $\sigma<0.127 \mathrm{keV}$ and the best-fitting line energy of $E_{\text {line }}$ $=6.44 \pm 0.04 \mathrm{keV}$ for this narrow emission line. The equivalent width of the line component is $51_{-22}^{+13} \mathrm{eV}$. The narrow emission line is at $6.44 \mathrm{keV}$ and can be interpreted as the neutral $\mathrm{Fe} \mathrm{K} \alpha$ emission line.

Based on the narrow neutral $\mathrm{Fe} \mathrm{K} \alpha$ emission line in the iron band, we model the broad-band spectrum with MODEL1. MODEL1 offers a very good fit with $C$-stat $/ \nu=205.35 / 161$. The best-fitting model and corresponding ratio plot are shown in Fig. A1. The relativistic disc 


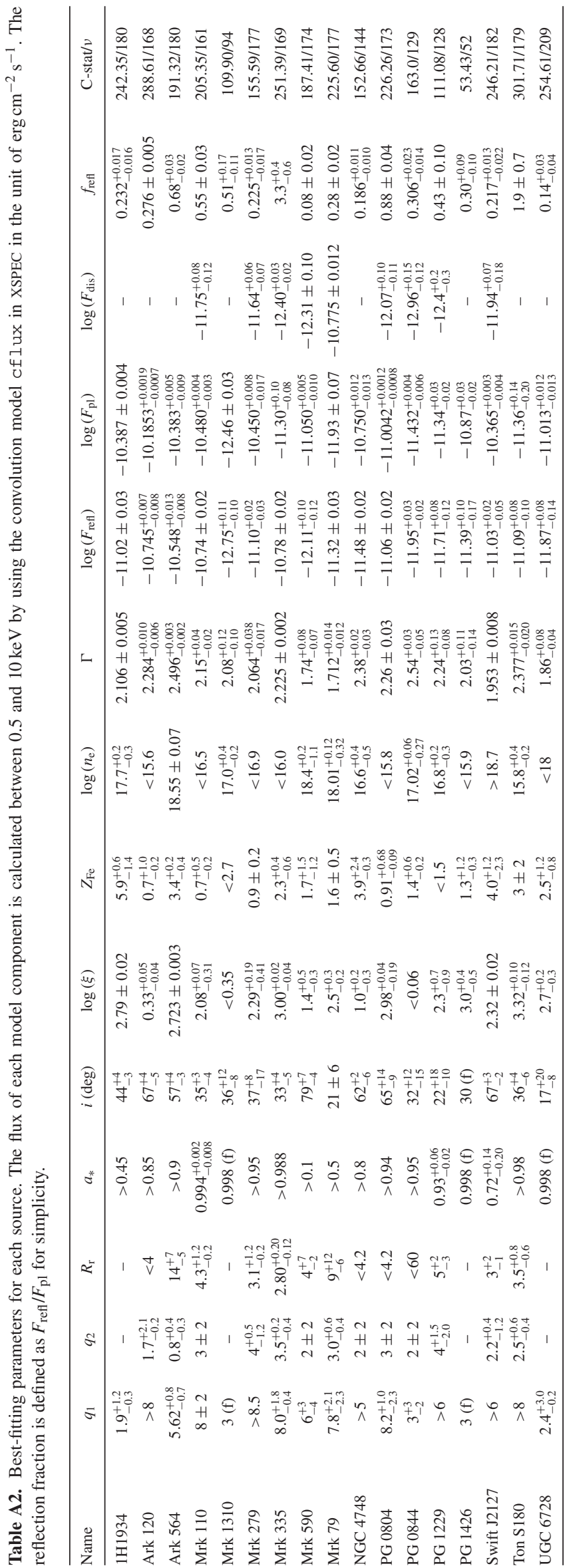


Table A3. Best-fitting model parameters for Ton S180 when the soft excess is modelled by a soft cutoff model and a fixed disc density $\log \left(n_{\mathrm{e}}\right)=15$ is assumed. $E_{\text {cut }}$ is in the unit of $\mathrm{keV}$. The units of other parameters are the same as in Table A2.

\begin{tabular}{lcc}
\hline Model & Parameter & Value \\
\hline cutoff & $\Gamma$ & $3.32_{-0.13}^{+0.12}$ \\
& $E_{\text {cut }}$ & $11_{-7}^{+32}$ \\
powerlaw & Norm & $1.8_{-0.6}^{+0.4} \times 10^{-3}$ \\
& $\Gamma$ & $2.04_{-0.13}^{+0.10}$ \\
relxilld & $\log \left(F_{\mathrm{pl}}\right)$ & $-11.34_{-0.37}^{+0.16}$ \\
& $q_{1}$ & $>8$ \\
& $q_{2}$ & $2.6_{-0.2}^{+0.4}$ \\
& $R_{\mathrm{r}}$ & $5_{-2}^{+3}$ \\
& $i$ & $38 \pm 2$ \\
& $a_{*}$ & $0_{-1}^{+1}$ \\
& $Z_{\mathrm{Fe}}$ & $>8$ \\
C-stat $/ v$ & $\log (\xi)$ & $3.0 \pm 0.1$ \\
\hline
\end{tabular}

reflection model accounts for mainly the soft excess below $2 \mathrm{keV}$. The lack of the broad $\mathrm{Fe} \mathrm{K} \alpha$ emission line in the iron band might be due to the extremely blurred reflection component, as seen in our modelling. Only an upper limit of the disc density $\left(\log \left(n_{\mathrm{e}}\right)<16.5\right)$ is found.

\section{A5 Mrk 1310}

Mrk 1310 is a Seyfert 1 galaxy (Véron-Cetty \& Véron 2006) and has only one XMM-Newton observation with a net pn exposure of $35 \mathrm{ks}$. The iron band does not show strong evidence for emission features. A power-law model can offer a very good fit for the spectra between 3 and $10 \mathrm{keV}$ with $\mathrm{C}$-stat $/ \nu=52.02 / 46$. By adding an additional line model zgauss with the line energy fixed at $6.4 \mathrm{keV}$, the fit can be improved by $\Delta \mathrm{C}$-stat $=4$ with two more free parameters. The equivalent width of the line component is $<20 \mathrm{eV}$. In the soft band, Mrk 1310 however shows a strong soft excess, as in other AGN in our sample.

We model the broad-band spectrum of Mrk 1310 with MODEL2. MODEL 2 offers a very good fit with C-stat $/ v=109.90 / 94$. The bestfitting model and corresponding ratio plot are shown in Fig. A1. The high density disc reflection component accounts for the soft excess emission. However, due to the lack of a broad $\mathrm{Fe} \mathrm{K} \alpha$ emission line, we are unable to constrain the disc emissivity profile and the spin of the $\mathrm{BH}$. We assume the emissivity index in a flat spacetime $\left(q_{1}=q_{2}\right.$ $=3$ ) and a maximum spin parameter. The best-fitting parameters are shown in Table A2. By modelling the soft excess emission with high density disc reflection model, a high disc density of $\log \left(n_{\mathrm{e}}\right)=17_{-0.2}^{+0.4}$ is required.

\section{A6 Mrk 279}

Previous analysis of the long XMM-Newton observations of the Seyfert 1 galaxy Mrk 279 (Véron-Cetty \& Véron 2006) in 2005 by Costantini et al. (2010) shows very complex emission features in the iron band, indicating both a broad $\mathrm{Fe} \mathrm{K} \alpha$ emission line from the disc and a narrow Fe XXVI line potentially from the outer layer of the torus.

A ratio plot of the stacked pn spectrum of Mrk 279 fitted with an absorbed power law is shown in Fig. 3. The iron band of the spectrum shows two narrow emission features and a broad line component. By modelling the line features with multiple zgauss models, we obtained a very good fit in the $3-10 \mathrm{keV}$ band. Three zgauss models are required: a broad line at $6.6_{-0.3}^{+0.5} \mathrm{keV}(\mathrm{EW}=$ $\left.50_{-13}^{+22} \mathrm{eV}, \sigma=0.38_{-0.08}^{+0.12} \mathrm{eV}\right)$; a narrow line at $6.41_{-0.04}^{+0.02} \mathrm{keV}(\mathrm{EW}$ $=85_{-12}^{+14} \mathrm{eV}, \sigma<0.02 \mathrm{eV}$ ); a second narrow line at $6.98_{-0.12}^{+0.06} \mathrm{keV}$ $(\mathrm{EW}=<22 \mathrm{eV}, \sigma<0.02 \mathrm{eV})$. The second narrow line at $6.98 \mathrm{keV}$ is consistent with previous analysis by Costantini et al. (2010). However, the line feature is too weak to be constrained with an unconstrained equivalent width and a small statistical improvement when the line model is added to the fit $(\Delta \mathrm{C}$-stat $=3$ with three more parameters).

Based on the indication of the iron band, we model the broadband spectrum of Mrk 279 with MODEL1. MODEL1 offers a very good fit with C-stat $/ \nu=155.59 / 177$. The best-fitting model is shown in Fig. A2. The relativistic disc reflection model accounts for both the soft excess and the broad Fe $\mathrm{K} \alpha$ emission line in Mrk 279. A high BH spin of $a_{*}>0.95$ is required for the spectral modelling and only an upper limit of the disc density $\log \left(n_{\mathrm{e}}\right)<16.9$ is achieved. We obtain a disc viewing angle of $i=37_{-17}^{+8}$, which is consistent with previous analysis $\left(i<30^{\circ}\right.$; Costantini et al. 2010).

\section{A7 Mrk 335}

Mrk 335 is an NLS1 (Véron-Cetty \& Véron 2006) that has been well studied in the X-ray band. This source experienced several extremely low flux states in history (e.g. Grupe, Komossa \& Gallo 2007; Parker et al. 2014). Grupe et al. (2012) found that the complex spectral variability can be explained by a variable disc reflection component. Parker et al. (2014) and Gallo et al. (2015) explain the low flux state spectrum of Mrk 335 with a reflection-dominated emission from the inner disc region. The spectral variability is due to strong light-bending effects in the vicinity of the central $\mathrm{BH}$. The strongest supporting evidence for the reflection interpretation of the spectrum of Mrk 335 is the discovery of the reverberation lag between the reflected disc photons and the coronal continuum photons (Kara et al. 2013).

We first fit the stacked spectrum of Mrk 335 with an absorbed power-law model and the ratio plot is shown in Fig. 3. The ratio plot shows a strong broad $\mathrm{Fe} \mathrm{K} \alpha$ emission line feature and a strong soft excess below $2 \mathrm{keV}$. The result is similar to Parker et al. (2014). By following the indication in Parker et al. (2014), we model the broad-band spectrum with MODEL4. MODEL4 offers a very good fit with C-stat $/ v=251.39 / 169$. The best-fitting model is shown in Fig. A2. One thin warm absorber modelled by warmabs with $N_{\mathrm{H}}=2.45_{-0.17}^{+0.39} \times 10^{20} \mathrm{~cm}^{-2}$ and $\log (\xi)=1.38 \pm 0.02$ is found. We obtain a very high reflection fraction $f_{\text {refl }}=3.3_{-0.6}^{+0.4}$, indicating a reflection-dominated scenario, similar with previous analysis (e.g. Parker et al. 2014). By fitting the broad Fe K $\alpha$ emission line and the soft excess with the same reflection model, we obtain a very steep disc emissivity profile (see Table A2 for best-fitting parameters). The large inner emissivity index $q_{1}$ and the low broken radius $R_{\mathrm{r}}$ indicate a very compact coronal region (e.g. $<3 r_{\mathrm{g}}$; Parker et al. 2014). Only an upper limit of the disc density $\log \left(n_{\mathrm{e}}\right)<16.0$ is achieved. We find a very BH spin of $a_{*}>0.988$ and a small inclination angle of $i=33_{-5}^{+4}$, which are consistent with previous analysis of NuSTAR observations of the same source (e.g. $a_{*} \approx$ $0.99, i \approx 25^{\circ}$; Parker et al. 2014). Our best-fitting inclination angle 

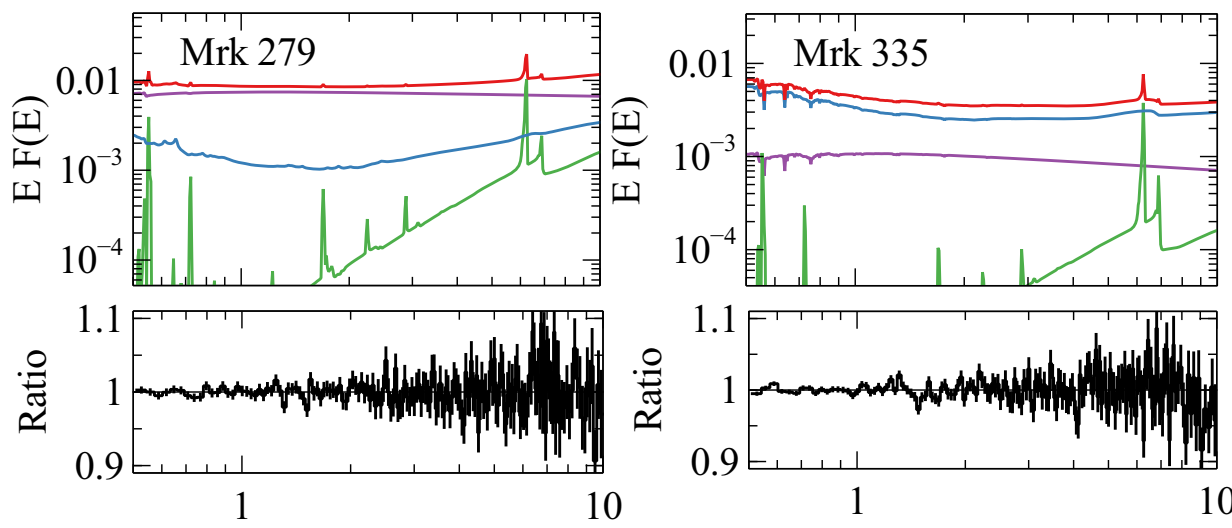

Energy $(\mathrm{keV})$

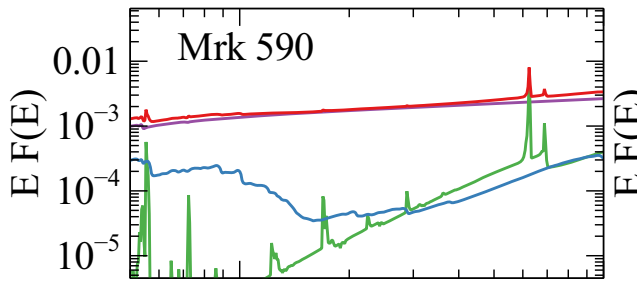

Energy $(\mathrm{keV})$
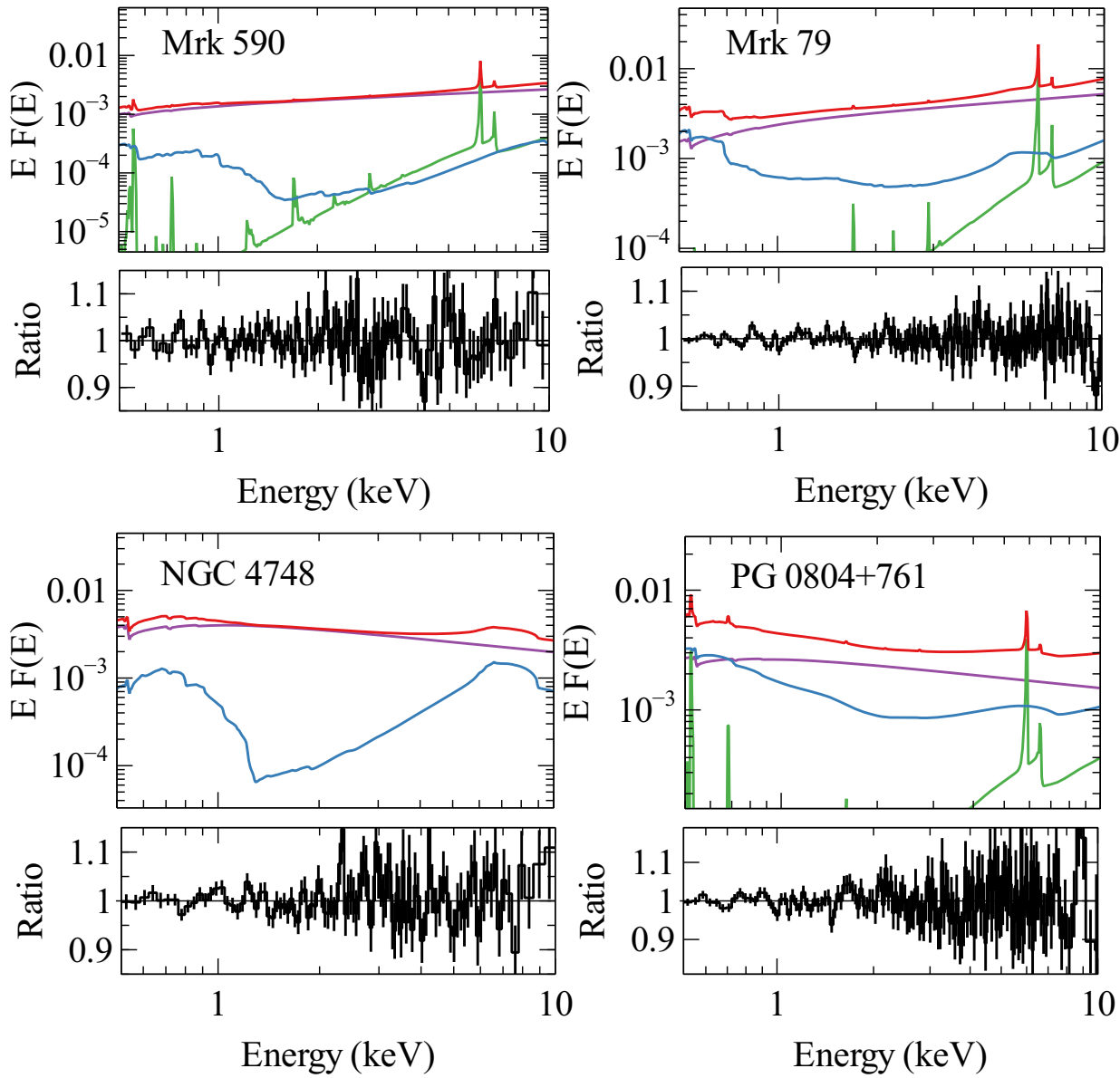

Figure A2. Fig. A1 continued.

is however lower than the value measured using Suzaku observations (e.g. $i \approx 50-58^{\circ}$; Walton et al. 2013; Gallo et al. 2015).

\section{A8 Mrk 590}

Mrk 590 is a Seyfert 1 galaxy (Véron-Cetty \& Véron 2006). Previous analysis of the quasi-simultaneous XMM-Newton and Chandra observations in 2004 shows evidence for a strong soft excess and narrow $\mathrm{Fe} \mathrm{K} \alpha, \mathrm{Fe} \mathrm{XXV}$, and Fe XXVI emissions (Longinotti et al. 2007).

We first fit the $3-10 \mathrm{keV}$ band spectrum with an absorbed powerlaw model and the ratio plot is shown in Fig. 3. By fitting the narrow lines features with simple zgauss models. Two narrow line models are required, one line at $6.407 \pm 0.02 \mathrm{keV}\left(\mathrm{EW}=135_{-23}^{+12} \mathrm{eV}, \sigma<\right.$ $0.06 \mathrm{keV})$ and the other line at $7.04_{-0.10}^{+0.06} \mathrm{keV}\left(\mathrm{EW}=46_{-35}^{+23}, \sigma<\right.$ $0.12 \mathrm{keV})$. The former line can be interpreted as $\mathrm{Fe} \mathrm{K} \alpha$ emission line and the latter can be interpreted as Fe XXVI line. We do not find strong evidence for a narrow Fe XXV emission line as in Longinotti et al. (2007) or a broad emission feature.

Based on the analysis of the iron band, we model the broad-band spectrum of Mrk 590 with MODEL1. MODEL1 can provide a very good fit with C-stat $/ \nu=187.41 / 174$. The best-fit model is shown in Fig. A2. The disc reflection component with a very high disc density of $\log \left(n_{\mathrm{e}}\right)=18.4_{-1.1}^{+0.2}$ accounts for the soft excess, as shown 
in Fig. A2. We obtain a lower limit of the BH spin of $a_{*}>0.1$ and a high inclination angle of $i=79_{-4}^{+7^{\circ}}$.

\section{A9 Mrk 79}

Mrk 79 is a Seyfert 1 galaxy (Véron-Cetty \& Véron 2006) that shows very large X-ray flux variability by a factor of 10 in multi-epoch observations (Gallo et al. 2011b). The spectrum of Mrk 79 shows a variable soft excess and strong narrow emission line features in the iron band.

We stack all the EPIC-pn spectra in the archive and find two narrow emission lines in the iron band. The first line is at $6.40 \pm 0.02 \mathrm{keV}$ in the source frame $\left(\mathrm{EW}=173_{-14}^{+32} \mathrm{eV}, \sigma=\right.$ $\left.0.086_{-0.020}^{+0.019} \mathrm{keV}\right)$ and the second line is at $6.90 \pm 0.05 \mathrm{keV}$ in the source frame $\left(\mathrm{EW}=30_{-13}^{+22} \mathrm{eV}, \sigma<0.08 \mathrm{keV}\right.$ ), corresponding to $\mathrm{Fe} \mathrm{K} \alpha$ and $\mathrm{Fe} \mathrm{K} \beta$ line.

Based on the analysis of the iron band, we model the broad-band spectrum of Mrk 79 with MODEL1. MODEL1 offers a very good fit with C-stat $/ \nu=225.60 / 177$. The best-fitting model is shown in Fig. A2. Mrk 79 has the hardest continuum emission in our sample with $\Gamma=1.712_{-0.012}^{+0.014}$. According to our calculation of the mass accretion rate using $\mathrm{B}$ band flux, Mrk 79 indeed has the lowest accretion rate in our sample $\left(\dot{m}=0.13_{-0.07}^{+0.05}\right)$. A high disc density of $\log \left(n_{\mathrm{e}}\right)=18.01_{-0.32}^{+0.12}$ is required by our spectral modelling. We obtain a BH spin of $a_{*}>0.5$ and an inclination angle of $i=21 \pm 6^{\circ}$, which is consistent with previous analyses $\left(a_{*} \approx 0.7, i \approx 21^{\circ}\right.$; Gallo et al. 2011a).

\section{A10 NGC 4748}

NGC 4748 is an NLS1 (Véron-Cetty \& Véron 2006) that is not well studied in the X-ray band. Only one XMM-Newton observation with a net pn exposure of $26 \mathrm{ks}$ is available in the archive.

The spectrum of NGC 4748 in the iron band shows a broad emission feature. See Fig. 3 for a ratio plot of the spectrum of NGC 4748 fitted by an absorbed power-law model. By fitting the line model with one zgauss model, the line width of the emission line is $\sigma=0.7_{-0.3}^{+0.6} \mathrm{keV}$ and the line is at $6.7_{-0.2}^{+0.3} \mathrm{keV}$. The equivalent width of the line is $373_{-17}^{+42} \mathrm{eV}$. This strong, broad $6.7 \mathrm{keV}$ emission line can be interpreted as the relativistic disc Fe K emission line. A simple zgauss modelling of the line feature leaves some residuals between 7 and $8 \mathrm{keV}$. No significant evidence for a narrow core is found.

Based on the evidence for a broad iron emission line feature and a soft excess, we model the broad-band spectrum with MODEL2. MODEL2 offers a very good fit with C-stat $/ \nu=152.66 / 144$. Fig. A2 shows the best-fitting model and the corresponding ratio plot. One relativistic reflection model is able to model both the soft excess mission and the broad iron line feature. An intermediate disc density is required $\log \left(n_{\mathrm{e}}\right)=16.6_{-0.5}^{+0.4}$. A high $\mathrm{BH}$ spin of $a_{*}>0.8$ is preferred by our model.

\section{A11 PG $0804+761$}

PG $0804+761$ is a Seyfert 1 galaxy (Véron-Cetty \& Véron 2006) and has three XMM-Newton observations in the archive. The first observation in 2000 (obsID 0102040401) was entirely dominated by flaring particle background. The other two observations were taken in 2010 and have a total net pn exposure of $32 \mathrm{ks}$. By fitting the stacked pn spectrum of PG $0804+761$ with an absorbed power-law model, two strong emission lines are shown in the iron band. A strong soft excess is found below $2 \mathrm{keV}$. See Fig. 3 for the ratio plot. We first model the line features with simple zgauss models. The line width of both emission line is $\sigma<0.08 \mathrm{keV}$, indicating two narrow lines from distant reflector. The first line is at $6.44 \pm 0.04 \mathrm{keV}\left(\mathrm{EW}=99_{-17}^{+35} \mathrm{eV}\right)$ and the second line is at $6.88_{-0.07}^{+0.06} \mathrm{keV}\left(\mathrm{EW}=91_{-24}^{+13} \mathrm{eV}\right)$. These two lines can be interpreted as $\mathrm{Fe} \mathrm{K} \alpha$ and $\mathrm{Fe} \mathrm{K} \beta$ lines. The spectrum shows no evidence for a broad line component.

Based on the evidence for only narrow Fe $\mathrm{K}$ emission lines, we model the broad-band spectrum with MODEL1. MODEL1 can offer a very good fit with C-stat $/ \nu=226.26 / 173$. The relativistic disc reflection model accounts for the soft excess. See Fig. A2 for the best-fitting model and corresponding ratio plot. Only an upper limit of the disc density $\log \left(n_{\mathrm{e}}\right)<15.8$ is found.

\section{A12 PG $0844+349$}

PG $0844+349$ is a Seyfert 1 galaxy (Véron-Cetty \& Véron 2006) that has shown a large flux variability of a factor of 10 in history. Gallo et al. (2011b) analysed the XMM-Newton observation taken during the X-ray weak state of PG $0844+349$ and found the spectrum is dominated by the disc reflection component, indicating strong light-bending effects.

We first fit the spectrum with an absorbed power-law model and the ratio plot is shown in Fig. 3. By fitting the emission features with two simple zgauss models, we obtain a broad line component at $6.6 \pm 0.2 \mathrm{keV}\left(\mathrm{EW}=349_{-24}^{+32} \mathrm{eV}, \sigma=0.31_{-0.13}^{+0.26} \mathrm{keV}\right)$ and a narrow core at $6.4 \mathrm{keV}(\mathrm{EW}<42 \mathrm{eV}, \sigma<0.05 \mathrm{keV})$. Both two line components are consistent with the results in Gallo et al. (2011b). However only an upper limit of the equivalent width of the second line is obtained due to a short net exposure of only $18 \mathrm{ks}$.

Based on the analysis in the iron band, we model the broad-band spectrum with MODEL1. MODEL1 can offer a very good fit with C$s t a t / v=163.0 / 129$. Fig. A3 presents the best-fitting MODEL 1 for PG $0844+349$. A disc reflection component with a high disc density parameter of $\log \left(n_{\mathrm{e}}\right)=17.2_{-0.27}^{+0.06}$ can account for both the broad iron line and the soft excess emission. We obtain a high BH spin of $a_{*}>0.95$ and an inclination angle of $i=32_{-15}^{+12^{\circ}}$. The inclination angle is consistent with the measurement in Gallo et al. (2011b, $i \approx 34^{\circ}$ ).

\section{A13 PG $1229+204$}

PG $1229+204$ is a Seyfert 1 galaxy (Véron-Cetty \& Véron 2006) and has only one XMM-Newton observation with a net pn exposure of only $17 \mathrm{ks}$. Fig. 3 shows a ratio plot of the EPIC-pn spectrum of PG $1229+204$ against an absorbed power-law model. The spectrum shows a strong soft excess below $2 \mathrm{keV}$ and an emission feature in the iron band. By fitting the emission line with one zgauss model, we find that the line central energy is $6.59_{-0.14}^{+0.15} \mathrm{keV}$ and the line width is $\sigma=0.23_{-0.11}^{+0.22} \mathrm{keV}$. The line feature is very strong with an equivalent width of $\mathrm{EW}=209_{-14}^{+23} \mathrm{eV}$. The short exposure of this observation does not allow us to distinguish a mildly broad emission feature or a combination of several emission lines (e.g. $\mathrm{Fe} \mathrm{K} \alpha, \mathrm{Fe} \mathrm{K} \beta$ ).

Because of the uncertainty of the nature of the emission feature in the iron band, we first fit the broad-band spectrum with MODEL2 to check if the relativistic disc reflection model is able to model both the soft excess emission and the emission feature in the iron band. MODEL2 can offer a good fit with C-stat $/ \nu=122.23 / 129$. However there are still residuals in the iron band indicating that the emission feature cannot be modelled with a relativistic disc reflection model. Secondly, we fit the spectrum with MODEL1, including a distant 

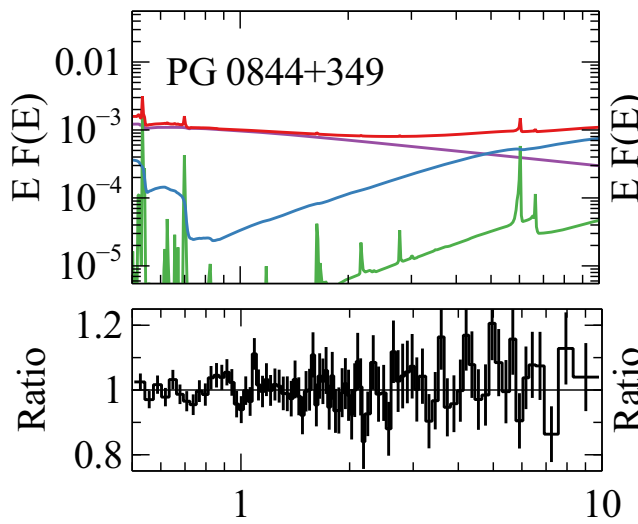

10
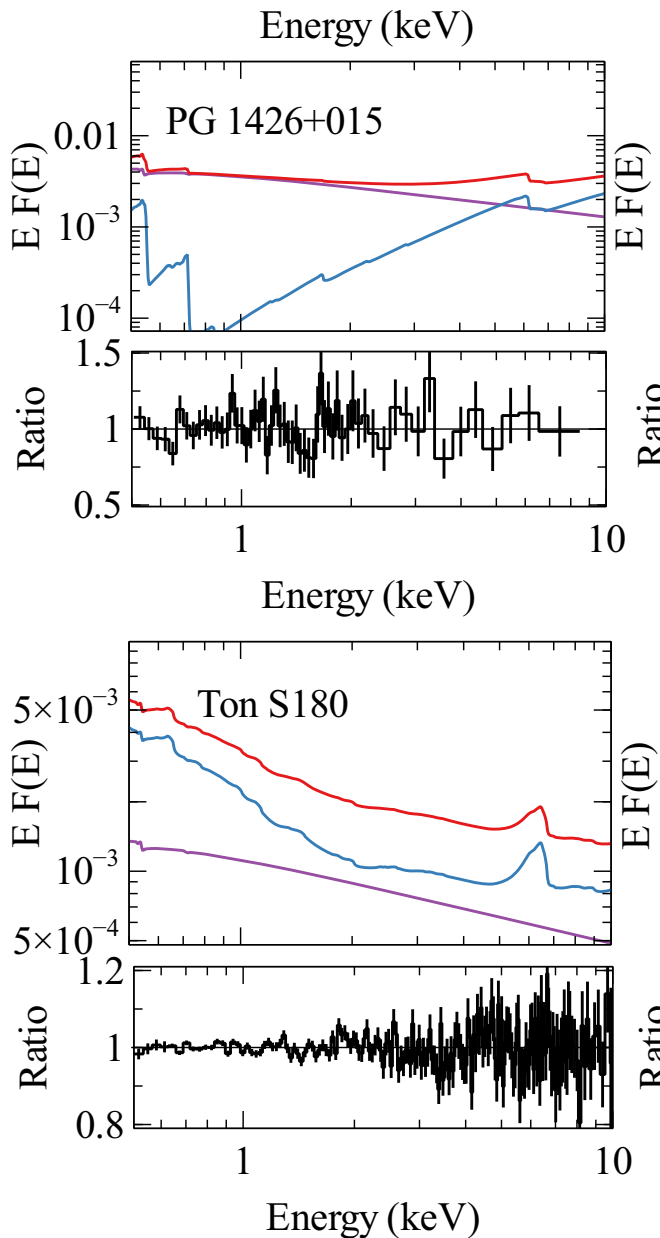
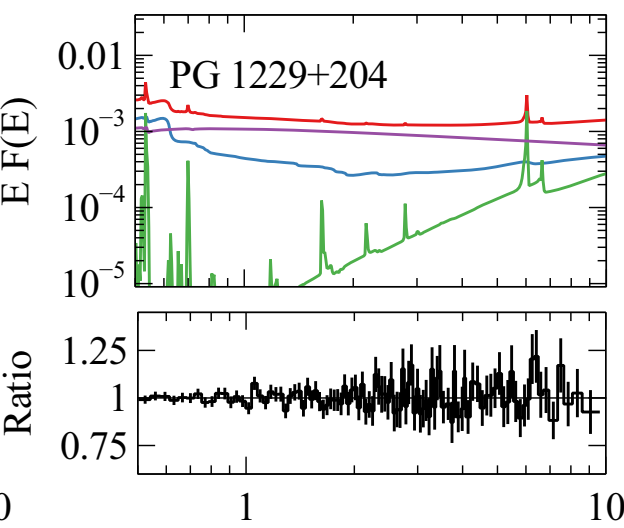

10

Energy $(\mathrm{keV})$
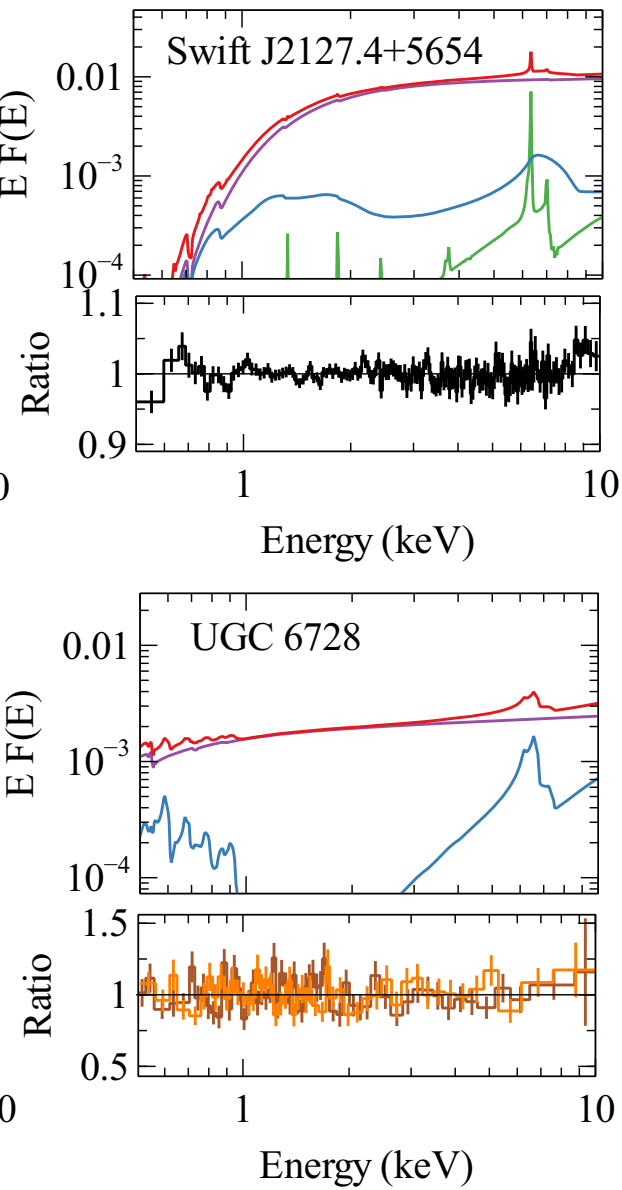

Figure A3. Fig. A2 continued.

neutral reflector. MODEL1 is able to improve the fit by $\Delta \mathrm{C}$-stat $=11$ with one more parameter with no structural residuals in the iron band. The best-fitting model and the corresponding ratio plot are shown in Fig. A3. The relativistic disc reflection component with a modest disc density of $\log \left(n_{\mathrm{e}}\right)=16.8_{-0.3}^{+0.2}$ accounts for the soft excess emission. We obtain a high BH spin of $a_{*}=0.93_{-0.02}^{+0.06}$ and an inclination angle of $i=22_{-10}^{+18^{\circ}}$.

\section{A14 PG 1426 + 015}

PG $1426+015$ is a Seyfert 1 galaxy (Véron-Cetty \& Véron 2006) and shows a blackbody-like soft excess emission (e.g. $\mathrm{kT} \approx 0.1 \mathrm{keV}$;
Page et al. 2004). Fig. 3 presents a ratio plot of PG $1426+015$ EPIC-pn spectrum fitted with an absorbed power-law model. The spectrum shows evidence for weak emission feature in the iron band and a strong soft excess emission. By fitting the emission feature of a zgauss model with a fixed line energy at $6.4 \mathrm{keV}$, we obtain an upper limit of the line width $\sigma<0.40 \mathrm{keV}$. Although the spectrum shows tentative evidence for a broad emission feature, the short exposure of the observation does not allow us to better constrain the line shape.

We model the broad-band spectrum of PG $1426+015$ with MODEL2. MODEL2 offers a very good fit with C-stat $/ \nu=53.43 / 52$. The best-fitting model is shown in Fig. A3. The emissivity profile, 
the $\mathrm{BH}$ spin, and the viewing angle are not constrained during our fit due to the short exposure. Therefore we fix the emissivity index at $q_{1}=q_{2}=3$, a maximum $\mathrm{BH}$ spin, and a viewing angle of $i=30^{\circ}$. We only obtain an upper limit of the disc density $\log \left(n_{\mathrm{e}}\right)<15.9$.

\section{A15 Swift J2127.4 + 5654}

Swift J2127.4 + 5654 is a Seyfert 1 galaxy (Véron-Cetty \& Véron 2006) that has been well studied in the X-ray band. Miniutti et al. (2009) analysed the Suzaku observations of this source and obtained a BH spin of $a_{*}=0.6 \pm 0.2$. This result has been confirmed by Marinucci et al. (2014) as well as where XMM-Newton observations are considered. A combination of broad $\mathrm{Fe} \mathrm{K} \alpha$ emission line and a narrow core is found in the iron band. The Suzaku spectrum of Swift J2127.4 + 5654 shows a blackbody-shaped soft excess Miniutti et al. (2009). Kara et al. (2015) analysed the NuSTAR and found a reverberation lag of both the iron line and the Compton hump, supporting the disc reflection interpretation of the broadband spectrum of this source.

By following the indication of Marinucci et al. (2014), we model the broad-band spectrum with MODEL4. The best-fitting model is shown in Fig. A3. An additional neutral absorber is required and the model ztbabs is used for this purpose. The redshift parameter of ztbabs is fixed at the value of the source. The best-fitting model is shown in Fig. A3. MODEL4 offers a good fit with C$\mathrm{stat} / \nu=246.21 / 182$. An intermediate spin of $a_{*}=0.72_{-0.20}^{+0.14}$ is found and is consistent with previous spin measurements (e.g. Miniutti et al. 2009; Marinucci et al. 2014). A higher inclination angle $(i=$ $67_{-2}^{+3^{\circ}}$ ) is found by fitting with a high density disc reflection model compared with previous analysis (e.g. $40^{\circ}$; Miniutti et al. 2009). A lower limit of the disc density $\left(\log \left(n_{\mathrm{e}}\right)>18.7\right)$ is obtained, indicating a potential high disc density.

\section{A16 Ton S180}

Ton S180 is an NLS1 galaxy (Véron-Cetty \& Véron 2006) that shows both a broad Fe $\mathrm{K}$ emission line and a strong soft excess (e.g. Vaughan et al. 2002; Takahashi, Hayashida \& Anabuki 2010; Nardini et al. 2012; Parker et al. 2018). Takahashi et al. (2010) demonstrate that the soft excess emission shown in the Suzaku spectrum of Ton S180 can be described as a disc blackbody-shaped model with a temperature of $\mathrm{kT}=0.075 \mathrm{keV}$. Nardini et al. (2012) found that the broad-band XMM-Newton and Suzaku spectra of Ton $\mathrm{S} 180$ can be modelled by a combination of two reflection components, one from the inner disc and one from a distant reflector. A more recent study by Parker et al. (2018) successfully model the broad-band XMM-Newton spectrum of Ton S180 with a combination of a soft Comptonization component, a hard Comptonization component from the corona, and a relativistic disc reflection component. The soft Compotonization component accounts for the soft excess. However the relativistic reflection component requires a very high iron abundance $Z_{\mathrm{Fe}}>9 \mathrm{Z}_{\odot}$.

We first fit the stacked EPIC-pn spectrum of Ton S180 with an absorbed power-law model. The corresponding ratio plot is shown in Fig. 3. Similar to previous analysis, a broad emission line in the iron band and a strong soft excess below $3 \mathrm{keV}$ are found in the spectrum. Secondly, we model the broad-band spectrum with MODEL2. MODEL2 offers a very good fit of both the broad emission line feature and the soft excess emission with C-stat $/ \nu=301.71 / 179$. The best-fitting model is shown in Fig. A3 and the best-fitting parameters can be found in Table A2. By modelling the broad line and the soft excess with the same model, we obtain a high $\mathrm{BH}$ spin of $a_{*}>0.98$. A very steep emissivity profile is found, indicating a very compact coronal region. A disc viewing angle of $36_{-6}^{+4^{\circ}}$ is found, which is consistent with the previous measurement (e.g. $\approx 39^{\circ}$; Parker et al. 2018). As shown from the plot of the best-fitting model, the spectrum is dominated by the disc reflection component with a reflection fraction $f_{\text {refl }}=1.9 \pm 0.7$, making it the second highest reflection fraction in our sample. A modest high disc density of $\log \left(n_{\mathrm{e}}\right)=15.6_{-0.2}^{+0.3}$ is required by spectral fitting.

\section{A17 UGC 6728}

UGC 6728 is a Seyfert 1 galaxy (Véron-Cetty \& Véron 2006) that has one of the known lowest black hole masses $\left(7.1 \times 10^{5} \mathrm{M}_{\odot}\right.$; Bentz \& Katz 2015). Only one XMM-Newton observation is available in the archive. The EPIC-pn observation of UGC 6728 is dominated by flaring particle background. Therefore, we use EPICMOS observations instead. The MOS spectra of UGC 6728 show tentative evidence for emission features in the iron band, similar with PG $1426+015$, and a strong soft excess below $2 \mathrm{keV}$.

We model the broad-band spectrum with MODEL2. The relativistic reflection model accounts mainly for the soft excess. The $\mathrm{BH}$ spin parameter is not constrained so we assume a maximum $\mathrm{BH}$ spin during the fit. MODEL2 offers a good fit with C-stat $/ \nu=254.61 / 209$. The best-fitting model is shown in Fig. A3. Only an upper limit of the disc density $\log \left(n_{\mathrm{e}}\right)<18$ is found. The best-fitting model predicts a very strong broad $\mathrm{Fe} \mathrm{K}$ emission line, which cannot be resolved by current data quality. More future observations with longer exposures will enable us to study the iron band of UGC 6728 in more details.

\section{APPENDIX B: THE DISC DENSITY OF A STABLE GAS PRESSURE-DOMINATED DISC}

In this section, we propose a possible explanation for the overestimation of the disc density in GX $339-4$ given by the classical disc theory: the disc of GX $339-4$ is gas dominated in the hard state while the Seyfert 1 AGN in our sample, which show a significantly higher accretion rate than GX $339-4$ in the hard state, are in the radiation pressure-dominated regime in the Shakura \& Sunyaev (1973, SS73) model. The disc density distribution across the height of a gas pressure-dominated disc might be the solution to this density problem in GX $339-4$ observations.

\section{B1 Gas pressure-dominated disc}

In this section, we introduce the disc behaviour at different accretion rates in the SS73 model considering gas and radiation pressure in the disc. We assume that a fraction $(f)$ of the disc energy is transferred to the coronal region instead of being radiated away locally from the disc (Svensson \& Zdziarski 1994). The critical condition for the disappearance of the radiation pressure-dominated region in the disc is given by $P_{\text {gas }}=P_{\text {rad }}$, where $P_{\text {gas }}$ and $P_{\text {rad }}$ are respectively the gas and the radiation pressure of the disc. According to Svensson $\&$ Zdziarski (1994), this condition at radius $r=R / R_{\mathrm{S}}=R / 2 r_{\mathrm{g}}$ leads to

$\frac{r}{\left(1-r^{-1 / 2}\right)^{16 / 21}}=188\left(\alpha m_{\mathrm{BH}} / 10^{8}\right)^{2 / 21} \dot{m}^{16 / 21}\left[\xi^{\prime}(1-f)\right]^{6 / 7}$,

$\alpha$ is the viscosity parameter and is assumed to be $0.1 ; M_{8}$ is $\mathrm{BH}$ mass in the unit $10^{8} \mathrm{M}_{\odot} ; \dot{m}$ is defined as $\dot{m}=\dot{M} c^{2} / L_{\mathrm{Edd}}=L_{\mathrm{Bol}} / \epsilon L_{\mathrm{Edd}}$; $\xi^{\prime}$ is the conversion factor in the radiative diffusion equation and 


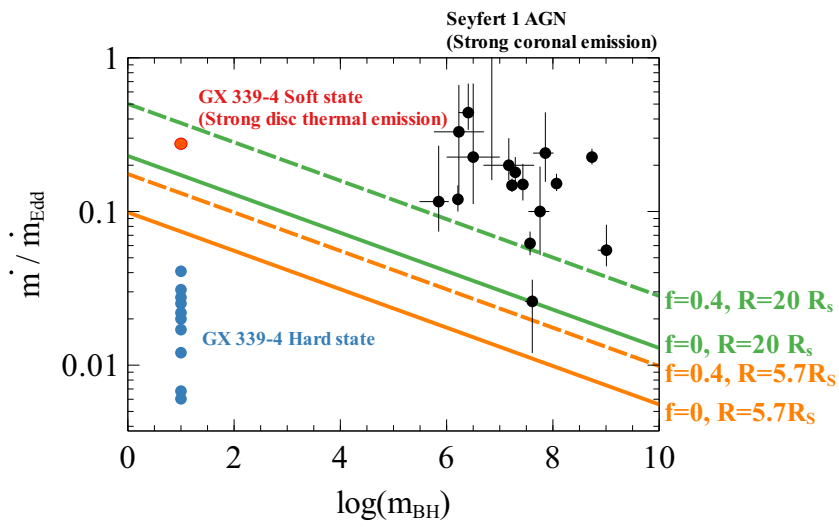

Figure B1. Mass accretion rates of 17 Seyfert 1 AGN and GX $339-4$ that have been studied in our series. The mass accretion rates of GX $339-4$ are calculated in our previous paper (Jiang et al. 2019b). The orange lines show the threshold mass accretion rate below which the whole disc is dominated by gas pressure. The green lines show the threshold mass accretion rate below which $R<20 R_{\mathrm{S}}$ is dominated by gas pressure. The solid lines are for $f=0$ is (SS73) and the dashed lines are for the scenario where 40 per cent of the disc energy is transferred to the coronal region (Svensson \& Zdziarski 1994).

assumed to be unity in the following calculation. When $f=0$, equation (B1) reproduces the result in SS73.

The left-hand side of equation (B1) has a minimum value when $r \approx 5.72$ (Svensson \& Zdziarski 1994). For certain $m_{\mathrm{BH}}$ and $f$, the right-hand side of equation (B1) is always smaller than the left-hand side when $\dot{m}$ is sufficiently small. In this case, there is no solution to equation (B1) and the radiation pressure-dominated region in the disc disappears. The orange lines of Fig. B1 show this threshold mass accretion rate for $f=0$ and $f=0.4$. For sources that are below the orange lines, the whole disc is dominated by gas pressure in the classical disc theory. For larger $\dot{m}$, the radiation pressuredominated region starts to expand from $r \approx 5.72$ both inwards and outwards in the disc. For example, the green lines of Fig. B1 show the threshold mass accretion rate below which $r>20$ is dominated by gas pressure.

The mass accretion rates of the 17 Seyfert 1 AGN in our sample and GX $339-4$ are also shown in Fig. B1. All Seyfery 1 AGN in our sample are above the green lines except Mrk 79. Mrk 79, which has a large uncertainty for the accretion rate estimation, is however still above the orange lines, indicating at least the inner region of the disc is dominated by radiation pressure. In comparison, the hard state of GX $339-4$ shows a much lower accretion rate and the corresponding observations indicate the source is in the gas pressure-dominated regime. The soft state of GX $339-4$ during an outburst however shows a much higher accretion rate and is the regime where the innermost region (e.g. $r \lesssim 20$ ) of the disc is dominated by radiation pressure. Note that the X-ray emission from GX $339-4$ is dominated by a strong disc thermal component with a relatively weak power-law continuum during this soft state observation (Parker et al. 2016; Jiang et al. 2019b) while the Seyfert $1 \mathrm{AGN}$ in our sample shows very strong power-law emission in the $\mathrm{X}$-ray band.

\section{B2 Disc density in a gas pressure-dominated disc}

So far, we have shown the indication that the accretion disc of GX $339-4$ in the hard state is in the gas pressure-dominated regime in the SS73 model. In this section, we calculate the disc density distribution across the height of a gas pressure-dominated disc in order to explain the density overestimation given by SS73.

SS73 and Svensson \& Zdziarski (1994) assume a thin disc with a uniform density across the height of a thin disc. The disc in reality however has a finite thickness which is particularly important for a gas pressure-dominated disc. Therefore, we calculate the surface disc density of a gas pressure-dominated disc assuming a simple isothermal disc model.

The standard thin disc model in SS73 gives the solutions of the number density $n_{\mathrm{e}}$ of a gas pressure-dominated disc:

$n_{\mathrm{e}}^{\text {gas }}=\frac{1}{\sigma_{\mathrm{T}} R_{\mathrm{S}}} K \alpha^{-7 / 10} r^{-33 / 20} \dot{m}^{2 / 5}\left[1-\left(r / r_{\text {in }}\right)^{-1 / 2}\right]^{2 / 5}$,

where $K=2^{-7 / 2}\left(\frac{512 \sqrt{2} \pi^{3}}{405}\right)^{3 / 10}\left(\alpha_{\mathrm{f}} \frac{m_{\mathrm{p}}}{m_{\mathrm{e}}}\right)^{9 / 10}\left(\frac{R_{\mathrm{S}}}{r_{\mathrm{e}}}\right)^{3 / 10} . \alpha_{\mathrm{f}}$ is the finestructure constant; $m_{\mathrm{p}}$ is the proton mass; $m_{\mathrm{e}}$ is the electron mass; $r_{\mathrm{e}}$ is the classical electron radius; $\sigma_{\mathrm{T}}=6.64 \times 10^{25} \mathrm{~cm}^{2}$ is the Thomson cross-section. This solution is shown by the red dashed line in Fig. 8 for $m_{\mathrm{BH}}=10$. The inferred disc densities in the corresponding observations are 100 times lower than the predictions in SS73 (see Fig. 8).

Here we estimate the disc density distribution in the disc that is regulated by gas pressure based on the following assumptions: (1) the total luminosity of the accretion process depends on the accretion rate at the mid-plane of the disc; (2) the solution of SS73 (equation B2) applies only to the mid-plane of the disc. Then we calculate the disc density within an optical depth of $\tau=1$, where the observed reflection spectrum comes from.

We ignore the self-gravitation of the disc. In the vertical direction of the disc, the pressure gradient is balanced by the gravitational force in the $z$ direction of the disc plane:

$\frac{\mathrm{d} P}{\mathrm{~d} z}=-\rho \frac{G M}{R^{3}} z$,

$P$ is the inner pressure of the disc; $M$ is the mass of the $\mathrm{BH}$.

For a gas-dominated disc, the inner pressure is

$\mathrm{d} P=C_{\mathrm{s}}^{2} \mathrm{~d} \rho$,

where $C_{\mathrm{s}}$ is the speed of sound and is about $6.3 \times 10^{4} \mathrm{~m} \mathrm{~s}^{-1}$ at $10^{7} \mathrm{~K}$ for the disc of a BH XRB.

By combining equations (B3) and (B4), we obtain

$\frac{\mathrm{d} \rho}{\rho}=-\frac{G M}{R^{3} C_{\mathrm{S}}^{2}} z \mathrm{~d} z$.

By integrating two sides, we obtain the disc density distribution of a gas-dominated disc:

$\ln \frac{n_{\mathrm{e}}}{n_{0}}=\ln \frac{\rho}{\rho_{0}}=-\frac{G M}{2 R^{3} C_{\mathrm{S}}^{2}} z^{2}$,

where $n_{0}$ and $\rho_{0}$ are the number density and the density at the midplane of the disc. We assume $n_{0} \approx n_{\mathrm{e}}^{\text {gas }}$ given in equation (B2) and a pure hydrogen disc. For a $10 \mathrm{M}_{\odot} \mathrm{BH}$ that is accreting at $\dot{m}=0.1$, the disc has a number density of $n_{0}=1.0 \times 10^{23} \mathrm{~cm}^{-3}$ and an equivalent density of $\rho_{0}=167 \mathrm{~kg} \mathrm{~m}^{-3}$ according to SS73 (see the red dashed line in Fig. 8). We define $J \equiv \frac{G M}{2 R^{3} C_{\mathrm{S}}^{2}}$ and then the number density is given by $n_{\mathrm{e}}=n_{0} e^{-J z^{2}}$. This density distribution in the vertical direction is consistent with equation (5.33) in Frank, King \& Raine (2002). Assuming $M=10 \mathrm{M}_{\odot}$ and $R=2 r_{\mathrm{g}}$ as in Fig. 8, the value of $J$ is approximately $6.22 \times 10^{-3} \mathrm{~m}^{-2}$.

Now we calculate the depth into the disc that is equivalent to the Thomson optical depth $\tau=1$. The optical depth is measured 
downwards from $+\infty$ to $z_{0}$,

$\tau=\int_{+\infty}^{z_{0}}-k \rho \mathrm{d} z=k \rho_{0} \int_{z_{0}}^{+\infty} e^{-J z^{2}} \mathrm{~d} z=\frac{k \rho_{0}}{\sqrt{J}} \int_{\sqrt{J} z_{0}}^{+\infty} e^{-x^{2}} \mathrm{~d} x$,

where $k$ is the opacity of pure hydrogen for free-electron scattering with the same efficiency at all wavelengths and equals $0.04 \mathrm{~m}^{2} \mathrm{~kg}^{-1}$. The right-hand side of equation (B7) should be equal to 1 . By solving the Gaussian integral in equation (B7), we obtain $\sqrt{J} z_{0}=1.76$ $\left(z_{0}=22 \mathrm{~m}\right)$. The density at $z_{0}$ is thus 4.5 per cent of the density in the mid-plane of the disc at $R=2 r_{\mathrm{g}}$. Similarly, we consider $R=5 r_{\mathrm{g}}$ and obtain $\sqrt{J} z_{0}=2.07$ and $n_{\mathrm{e}}=1.3$ per cent $n_{0}$. These fractions match the difference between our measurements and the solutions in SS73.

This paper has been typeset from a $\mathrm{T}_{\mathrm{E}} \mathrm{X} / \mathrm{LAT}_{\mathrm{E}} \mathrm{X}$ file prepared by the author. 